\title{
Atypical cadherin FAT4 orchestrates lymphatic endothelial cell polarity in response to flow
}

\author{
Kelly L. Betterman, ${ }^{1,2}$ Drew L. Sutton, ${ }^{1,2}$ Genevieve A. Secker, ${ }^{1,2}$ Jan Kazenwadel, ${ }^{1,2}$ Anna Oszmiana, ${ }^{1,2}$ Lillian Lim, ${ }^{3}$ Naoyuki Miura, ${ }^{4}$ \\ Lydia Sorokin, ${ }^{5}$ Benjamin M. Hogan, ${ }^{6,78}$ Mark L. Kahn, ${ }^{3}$ Helen McNeill, ${ }^{9,10,11}$ and Natasha L. Harvey ${ }^{1,2}$ \\ 'Centre for Cancer Biology, University of South Australia and SA Pathology, Adelaide, South Australia, Australia. ${ }^{2}$ SA Pathology, Adelaide, South Australia, Australia. ${ }^{3}$ Department of Medicine and \\ Cardiovascular Institute, University of Pennsylvania, Philadelphia, Pennsylvania, USA. ${ }^{4}$ Department of Biochemistry, Hamamatsu University School of Medicine, Hamamatsu, Japan. Innstitute of \\ Physiological Chemistry and Pathobiochemistry, University of Muenster, Muenster, Germany. ${ }^{6}$ Division of Genomics of Development and Disease, Institute for Molecular Bioscience, University of Queensland, \\ Saint Lucia, Queensland, Australia. 'Organogenesis and Cancer Program, Peter MacCallum Cancer Centre, Melbourne, Victoria, Australia. ${ }^{8}$ Department of Anatomy and Neuroscience, University of Melbourne, \\ Melbourne, Victoria, Australia. ' ${ }^{2}$ unenfeld-Tanenbaum Research Institute, Mount Sinai Hospital, Toronto, Ontario, Canada. ${ }^{10} \mathrm{Department}$ of Molecular Cenetics, University of Toronto, Toronto, Ontario, \\ Canada. "Department of Developmental Biology, Washington University School of Medicine, Saint Louis, Missouri, USA.
}

\begin{abstract}
The atypical cadherin FAT4 has established roles in the regulation of planar cell polarity and Hippo pathway signaling that are cell context dependent. The recent identification of FAT4 mutations in Hennekam syndrome, features of which include lymphedema, lymphangiectasia, and mental retardation, uncovered an important role for FAT4 in the lymphatic vasculature. Hennekam syndrome is also caused by mutations in collagen and calcium binding EGF domains 1 (CCBE1) and ADAM metallopeptidase with thrombospondin type 1 motif 3 (ADAMTS3), encoding a matrix protein and protease, respectively, that regulate activity of the key prolymphangiogenic VEGF-C/VECFR3 signaling axis by facilitating the proteolytic cleavage and activation of VECF-C. The fact that FAT4, CCBE1, and ADAMTS3 mutations underlie Hennekam syndrome suggested that all 3 genes might function in a common pathway. We identified FAT4 as a target gene of GATAbinding protein 2 (GATA2), a key transcriptional regulator of lymphatic vascular development and, in particular, lymphatic vessel valve development. Here, we demonstrate that FAT4 functions in a lymphatic endothelial cell-autonomous manner to control cell polarity in response to flow and is required for lymphatic vessel morphogenesis throughout development. Our data reveal a crucial role for FAT4 in lymphangiogenesis and shed light on the mechanistic basis by which FAT4 mutations underlie a human lymphedema syndrome.
\end{abstract}

\section{Introduction}

Hennekam syndrome (OMIM \#235510 and \#616006) is an autosomal-recessive disorder characterized by congenital lymphedema and lymphangiectasia, unusual facial morphology, attributed at least in part to intrauterine facial lymphedema, and a variable degree of intellectual disability (1-3). Approximately $25 \%$ of patients with Hennekam syndrome have been found to have mutations in CCBE1 (4), encoding collagen and calcium binding EGF domains 1, a secreted matrix protein that facilitates the proteolytic cleavage and activation of VEGF-C (5-7). ADAM metallopeptidase with thrombospondin type 1 motif 3 (ADAMTS3) cooperates with CCBE1 by proteolytically cleaving and activating VEGF-C (8), and mutations in ADAMTS3 were recently shown to underlie Hennekam syndrome (9). The VEGF-C/VEGFR3 signaling axis is of paramount importance for lymphangiogenesis $(10,11)$; VEGF-C is crucial for the exit and guidance of lymphatic endothelial progenitor cells from the embryonic veins to form the

Authorship note: KLB and DLS share co-first authorship. Conflict of interest: The authors have declared that no conflict of interest exists. Copyright: () 2020, American Society for Clinical Investigation.

Submitted: December 11, 2017; Accepted: March 5, 2020; Published: May 18, 2020 Reference information: J Clin Invest. 2020;130(6):3315-3328.

https://doi.org/10.1172/JCI99027. initial lymphatic vascular plexus (12), and VEGFR3, expressed by lymphatic endothelial cells (LECs), transduces VEGF-C-initiated signals to mediate LEC migration and proliferation $(13,14)$. Heterozygous loss-of-function mutations in both $\operatorname{VEGFC}(15,16)$ and Fms-related receptor tyrosine kinase 4 (FLT4), encoding VEGFR3 (12, 17-19), underlie human hereditary lymphedema, and mutations in genes involved in the VEGFR3 signaling pathway have been estimated to cause approximately $36 \%$ of primary lymphedema cases (20). Recently, FAT4 mutations were documented in patients with Hennekam syndrome in whom no CCBE1 mutations were detected (21), a discovery that uncovered an important role for FAT4 in the lymphatic vasculature. Moreover, this discovery suggested that FAT4, CCBE1, and ADAMTS3 might be components of the same signaling pathway.

FAT4 is the closest vertebrate homolog of Drosophila fat $(f t)$ $(22,23)$, an extremely large, atypical cadherin with roles in the control of planar cell polarity (PCP) and regulation of Hippo pathway signaling (24). These roles appear largely conserved in mammalian Fat4, although they are tissue dependent; Fat4 ${ }^{-/-}$mice die soon after birth and exhibit defective PCP in tissues including kidney, inner ear, neural tube (25), facial branchiomotor neurons (26), and sternum (27), whereas aberrant Hippo pathway activity, leading to elevated cell proliferation, has been reported in Fat4-deficient nephron progenitor cells (28), embryonic neuroepithelium (29), 

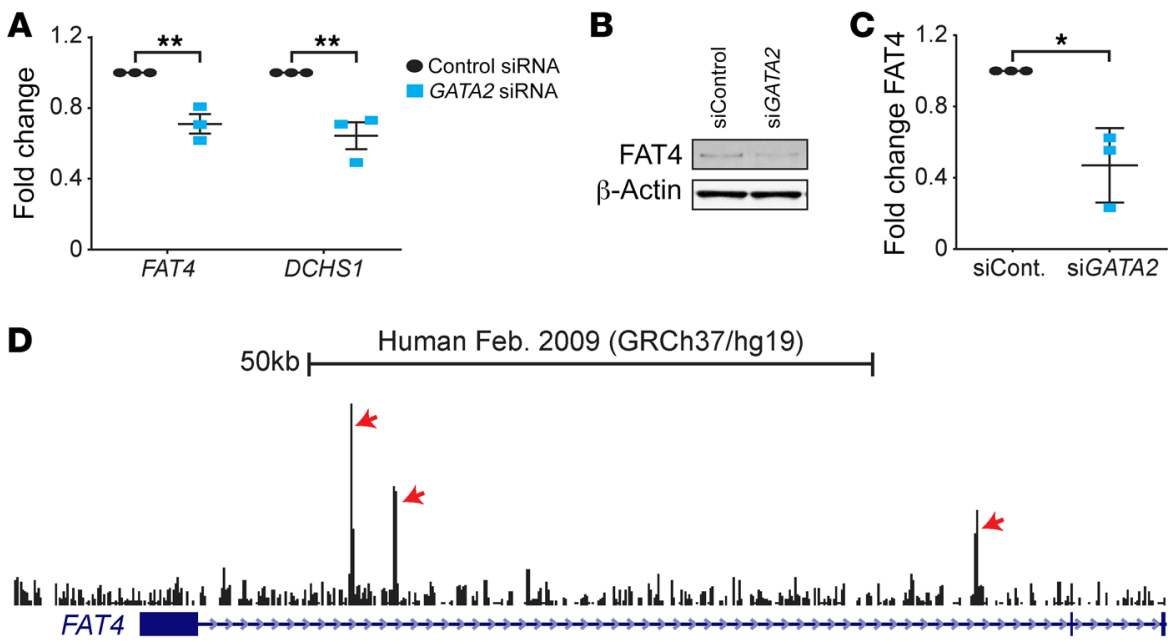

Figure 1. FAT4 and DCHS1 are GATA2 target genes. (A) GATA2-deficient hLECs had reduced expression of FAT4 and DCHS1 mRNA. (B) Western blots probed with FAT4 and $\beta$-actin revealed decreased FAT4 protein levels in hLECs treated with GATA2 siRNA (siGATA2). (C) Quantification of FAT4 levels following GATA2 knockdown. siCont., control siRNA. (D) ChIP-Seq profile demonstrating GATA2 occupancy (red arrows) in the first intron of FAT4 in hLECs. Data indicate the mean \pm SEM (A) or \pm SD (C). $n=3 .{ }^{*} P<0.05$ and ${ }^{* *} P<0.01$ by 2 -tailed Student's $t$ test. and heart (30). The appropriate allocation of cell polarity is a crucial determinant of ordered tissue architecture. Establishment of cell polarity within the vasculature is imperative both for the coordinated response of endothelial cells to sprouting and guidance cues that orchestrate vascular growth and for the specification of luminal and abluminal identity required to mediate vessel perfusion and endothelial cell barrier function. FAT4 has been demonstrated to coordinate cell polarity via interactions with its ligand, the large, atypical cadherin Dachsous1 (DCHS1) (31), a homolog of Drosophila Dachsous (23), and also genetically interacts with members of the "core" PCP pathway including VANGL planar cell polarity 2) (25). Intriguingly, Vangl2 and an additional core PCP component cadherin, EGF LAG seven-pass G-type receptor 1 (Celsr1) are both important for mediating the junctional remodeling and changes in cell orientation required for lymphatic vessel valve morphogenesis (32), and a mutation in CELSR1 was recently reported in hereditary lymphedema (33). Although less is known regarding the mechanisms by which FAT4 regulates mammalian Hippo pathway activity, FAT4 has been suggested to restrict access of the key transcriptional Hippo pathway effector yes-associated protein (YAP) to the nucleus, such that loss of Fat4 function facilitates the nuclear entry of YAP, resulting in elevated cell proliferation (28-30).

In a search for target genes of GATA binding protein 2 (GATA2), a key regulator of lymphatic vessel morphogenesis and valve development, in primary embryonic mouse LECs (mLECs), we identified Fat4 and Dchs1. Both genes were significantly reduced in expression following GATA2 knockdown in primary embryonic mLECs. Here, we describe a crucial cell-autonomous role for Fat4 in the control of LEC polarity during lymphatic vascular development. Our data reveal important roles for Fat4 in the regulation of polarity during lymphatic vessel morphogenesis and provide insight into the mechanisms by which loss-of-function mutations in FAT4 cause Hennekam syndrome.

\section{Results}

Fat4 and Dchs1 are GATA2 target genes in LECs. To identify genes regulated by the pivotal transcriptional regulator of lymphatic vessel valve morphogenesis, GATA2, we undertook gene profiling of control and Gata2 siRNA-treated primary mouse embry- onic LECs (mLECs). Analysis of the genes most significantly decreased in expression revealed that both Fat4 and Dchs1, encoding the large atypical cadherins FAT4 and DCHS1, were substantially decreased upon GATA2 knockdown. We next confirmed that FAT4 and DCHS1 were reduced in GATA2 siRNA-treated primary human LECs (hLECs); as expected, expression of both genes was decreased (Figure 1A). In the case of FAT4, this was also confirmed at the protein level, as FAT4 expression was reduced by approximately 55\% in GATA2-deficient cells (Figure 1, B and C). Analysis of the genome-wide binding profile of GATA2 in hLECs revealed that FAT4 is probably directly regulated by GATA2; prominent GATA2 binding peaks were identified within the first intron of FAT4 (Figure 1D). Given the recent description of FAT4 mutations in Hennekam syndrome (21), features of which include lymphedema and lymphangiectasia (1), we focused on defining the role of Fat4 in lymphatic vascular development.

Fat4 is important for lymphatic vessel morphogenesis during development. Fat4-deficient mice have been reported to die soon after birth and exhibit features consistent with disrupted PCP signaling including cystic kidneys, a shortened body axis, and aberrant inner ear hair cell organization (25). To determine whether Fat4 is important for lymphatic vascular development, we investigated lymphangiogenesis throughout development in Fat4 ${ }^{4 / 4}$ mice, generated by crossing Fat $4^{f / f l}$ mice (25) with $C M V$-Cre mice (34). Analysis of Fat $4^{4 / 4}$ embryos at E14.5 revealed that, in contrast to their littermate controls (Figure 2A), the majority of homozygous Fat4-deficient embryos (30 of 45 across 27 litters) exhibited subcutaneous edema (Figure 2D, arrow), suggestive of a lymphatic vascular defect. Whole-mount immunostaining of embryonic dorsal skin demonstrated a striking increase in lymphatic vessel width in E14.5 Fat4 ${ }^{4 / \Delta}$ embryos compared with lymphatic vessel widths in littermate controls (Figure 2, B, C, and E), coupled with a significant reduction in the number of lymphatic vessel branches (Figure $2 \mathrm{~F}$ ). No substantial changes in the caliber or branching of the dermal blood vascular network were observed (Supplemental Figure 1; supplemental material available online with this article; https:// doi.org/10.1172/JCI99027DS1). We next investigated lymphatic vessel morphology at E18.5, a stage at which valve development has been initiated in the collecting lymphatic vessels of the mouse 

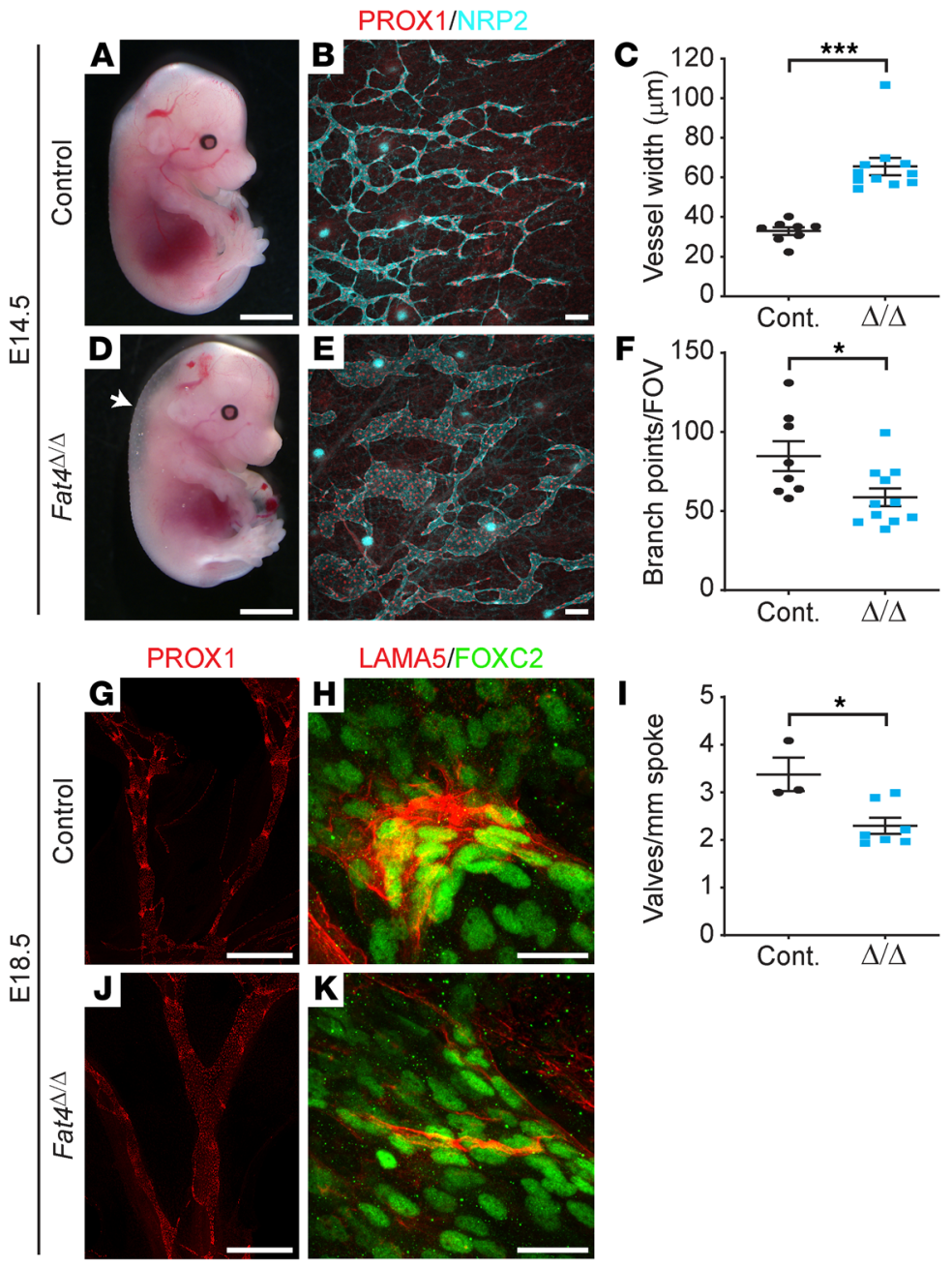

LAMA5/FOXC2
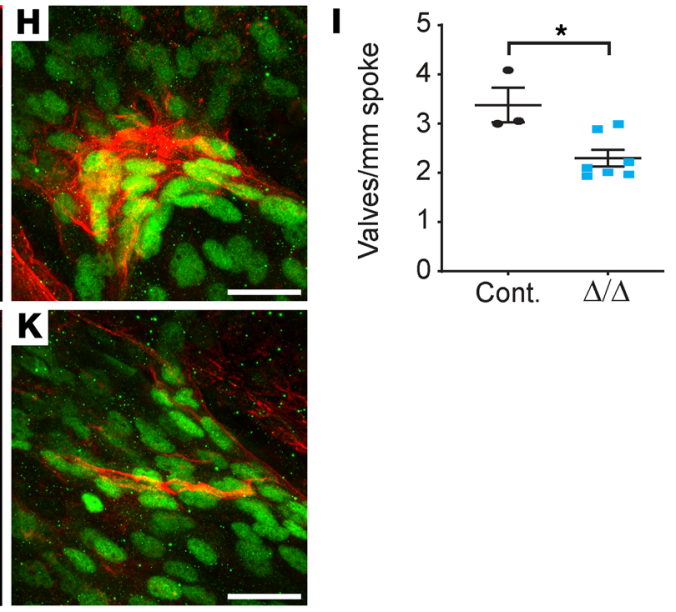

mesentery. We observed a significant reduction in the number of lymphatic vessel valves, indicated by clusters of PROX $1^{\text {hi }}$ cells, in $\mathrm{Fat}^{4 / \Delta}$ embryos compared with their control counterparts (Figure 2, G, I, and J). Moreover, immunostaining for laminin $\alpha 5$, a marker reflective of lymphatic vessel valve maturation (35), indicated that the valves that had initiated development were substantially less mature in Fat $4^{4 / 4}$ embryos than were those in control littermates (Figure 2, $\mathrm{H}$ and $\mathrm{K}$ ). Analysis of tissue sections revealed profound dilation of both dermal and submucosal lymphatic vessels in E18.5 $\mathrm{Fat}^{4 / \Delta}$ embryos compared with tissue sections from littermate controls (Supplemental Figure 2).

A LEC-autonomous requirement for Fat4 in lymphatic vessel morphogenesis. To address the LEC-autonomous requirement for Fat4 in lymphatic vascular development, we deleted Fat4 in the lymphatic vasculature by crossing Fat $4^{\text {fl/fl }}$ mice (25) with Prox1$C r e^{E R T 2}$ mice (36). We first analyzed dermal lymphangiogenesis at E14.5, following tamoxifen administration to pregnant females at E10.5, 11.5, and 12.5 (Figure 3, A-G). As observed in Fat4 $4^{4 / 4}$ embryos, the dermal lymphatic vessels of Fat $4^{\triangle L E C}$ embryos were significantly wider in caliber than were those of their control counterparts $\left(F a t 4^{f / f l}\right)$ (Figure 3, B, C, and E). Although there was a trend toward a reduced number of lymphatic vessel branches in Fat $4^{\triangle L E C}$ embryos, this decrease was not statistically significant (Figure $3 F$ ). In addition, subcutaneous edema was not gener-
Figure 2. Fat4 ${ }^{4 / 4}$ embryos exhibit lymphatic vessel defects. E14.5 Fat $4^{4 / 4}$ embryos exhibit subcutaneous edema (arrow, D) not evident in the littermate controls (A). Whole-mount immunostaining of the dorsal skin sprouting front revealed that dermal lymphatic vessels, stained with PROX1 (red) and NRP2 (cyan), were wider (B, C, and $\mathbf{E}$ ) and less branched (B, E, and $\mathbf{F})$ in E14.5 Fat4 ${ }^{4 / 4}$ embryos $(\Delta / \Delta)$ compared with littermate controls. Whole-mount immunostaining of E18.5 mesenteries with PROX1 (red) demonstrated reduced numbers of PROX1hi valves (per millimeter of spoke length) in Fat $4^{4 / \Delta}$ mesenteric lymphatic vessels (G, J, and I). Fat4 ${ }^{/ / 4}$ mesenteric lymphatic valve-forming cells ( $F O X C 2^{\text {hi }}$ cells, green) failed to polarize and had less laminin- $\alpha 5$ (LAMA5, red) than did littermate controls ( $\mathbf{H}$ and $\mathbf{K}$ ). Data indicate the mean \pm SEM. $n=8$ control embryos and $n=11$ $\Delta / \Delta$ embryos ( 7 independent E14.5 litters) ( $\mathbf{C}$ and $\mathbf{F}$ ); $n=3$ control embryos and $n=7$ Fat $^{4 / 4}$ embryos (3 independent E18.5 litters) (I). ${ }^{*} P<0.05$ and ${ }^{* *} P<0.0001$, by 2 -tailed Student's $t$ test. Scale bars: $2.5 \mathrm{~mm}$ (A and $\mathbf{D}), 100 \mu \mathrm{m}$ (B and $\mathbf{E}), 500 \mu \mathrm{m}$ (G and $\mathbf{J})$, and $25 \mu \mathrm{m}$ (H and $\mathbf{K})$. Cont., control.

ally observed in Fat4 ${ }^{\triangle L E C}$ embryos at E14.5 (Figure 3D). Together, these data suggested that potentially additional, non-LEC-autonomous roles of Fat4 might contribute to the branching and edema phenotypes that are a feature of $\mathrm{Fat}^{4 / \Delta}$ embryos. To investigate this possibility, we documented the expression of Fat4 with respect to the embryonic lymphatic vasculature using RNA ISH. These analyses revealed prominent Fat4 expression in the mesenchyme surrounding lymphatic vessels, as well as in the heart, particularly in cardiac valves, (Supplemental Figure 3), where both Dchs1 and Fat4 play important morphogenetic roles $(31,37,38)$. Mesenchymal FAT4 could potentially affect branching of the dermal lymphatic vasculature, either via homotypic interaction with FAT4 in LECs, or heterotypic interaction with DCHS1 in LECs, whereas cardiac defects are commonly reported to cause edema. Together, these expression data provide potential mechanisms to explain the presence of the branching and edema phenotypes in $\mathrm{Fat}^{4 / \Delta}$ but not Fat $4^{\triangle L E C}$ embryos.

We next analyzed mesenteric lymphatic vessel valve development in Fat $4^{\triangle L E C}$ embryos at E18.5, following the administration of tamoxifen at E12.5, 13.5, and 14.5 (Figure 3, H-M). Whole-mount immunostaining of mesenteric collecting lymphatic vessels revealed that, like Fat $4^{4 / \Delta}$ embryos, the number of PROX1 ${ }^{\text {hi }}$ lymphatic vessel valve territories was significantly reduced in Fat $4^{\triangle L E C}$ embryos compared with that observed in the control counterparts (Figure 3, H, J, and K). As in Fat $4^{4 / \Delta}$ embryos, lymphatic vessel valves also appeared less mature in Fat $4^{\triangle L E C}$ mice, reflected by greatly reduced laminin $\alpha 5$ levels in prospective valve territories of mutants compared with levels in controls (Figure 3, I and L). These data demonstrate that Fat4 regulates lymphatic vessel growth and morphogenesis, and in particular valve development, in a LEC-autonomous manner.

Fat4 controls LEC polarity. In considering the mechanisms underlying the dramatic lymphatic vascular phenotypes observed in Fat4-deficient embryos, we assessed the integrity of 2 pathways regulated by Fat4 in a tissue-specific context: cell polarity (25-27) and Hippo (28-30) pathway activity. Given the similarity of the der- 

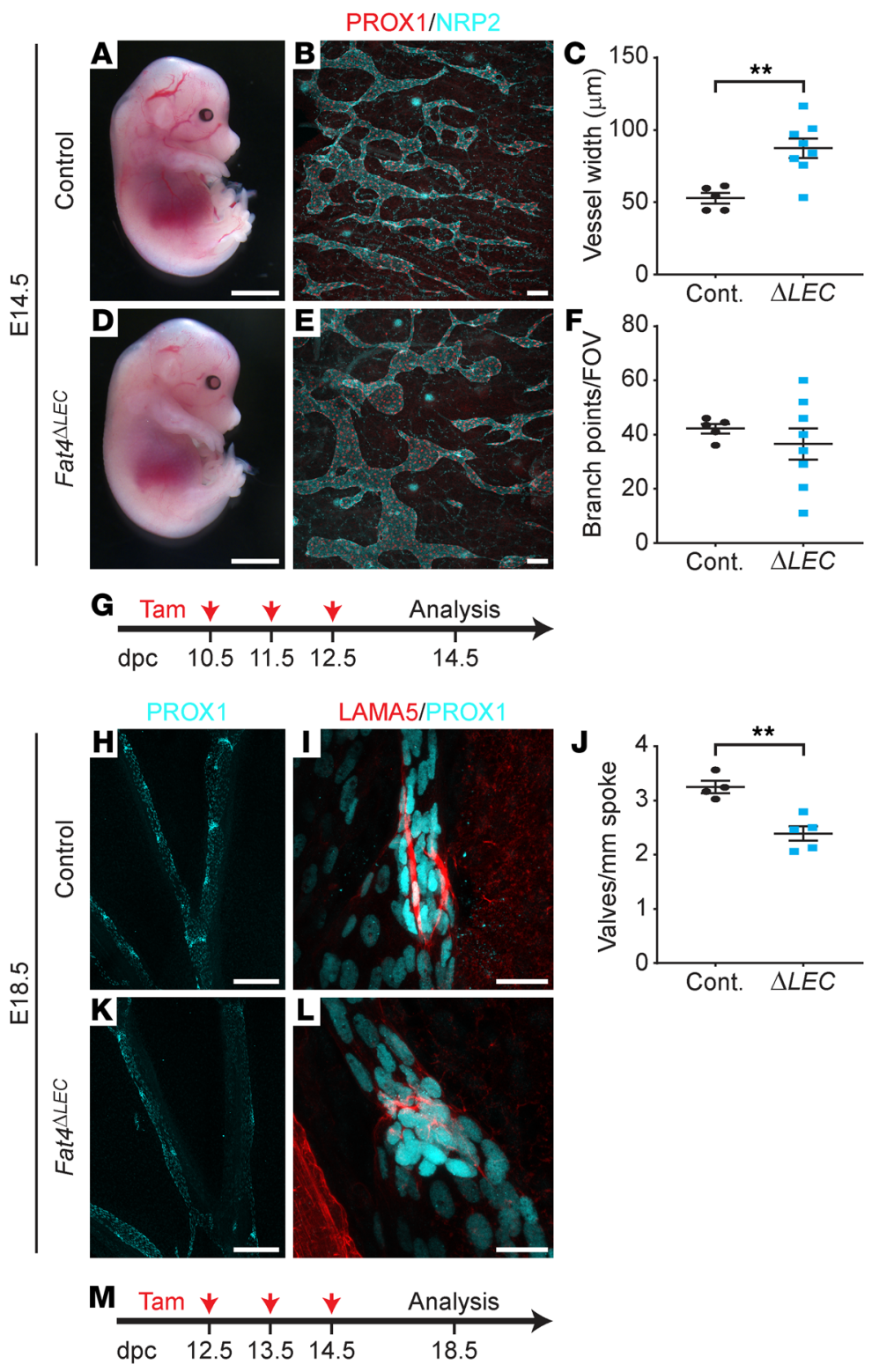

mal lymphatic vascular phenotype of Fat4-deficient mice to that of Pkd1-mutant mice, which exhibit striking defects in cell polarity (39), we first investigated polarity within the lymphatic vasculature of $\mathrm{Fat}^{4 / \Delta}$ embryos. We used 2 measures to assess cell polarity: Golgi position in sprouting dermal lymphatic vessels and nuclear ellipticity. We found that the Golgi, usually positioned ahead of the nucleus in the direction of dermal lymphatic vessel sprouting toward the embryonic midline (39), was more randomly positioned in E14.5 Fat $4^{\Delta / \Delta}$ mice compared with their control counterparts (Figure 4, A-D). Likewise, nuclear morphology and overall cell shape, normally elliptical in actively migrating cells and more rounded in static cells (39), were altered in E14.5 Fat4 mutants; cells were more rounded (Figure 4, E-H) and nuclei less elliptical (measured by the nuclear length/width ratio) in Fat $4^{4 / \Delta}$ mice compared with control mice (Figure 4I). Both of these measures indicated that the polarity of Fat4-deficient LECs was aberrant. To determine whether altered LEC polarity was a feature of Fat $4^{4 / \Delta}$ embryos from the onset of lymphatic vascular development, we examined the polarity of lym-
Figure 3. LEC-autonomous requirement for Fat4 in lymphatic vessel morphogenesis. Prox1-CreERT2 Fat $4^{f / / f l}$ male mice were crossed with Fat $4^{f / f l}$ females, and tamoxifen $(20 \mathrm{mg} / \mathrm{mL}$, red arrows) was administered to pregnant females intraperitoneally at $10.5,11.5$, and 12.5 days postcoitum (dpc) (G), or 12.5, 13.5, and 14.5 dpc (M). Embryos were analyzed at E14.5 (A-F) and E18.5 (H-L). E14.5 Fat4 ${ }^{4 L E C}$ embryos appeared phenotypically normal (A and D). Whole-mount immunostaining of the dorsal skin sprouting front revealed that the dermal lymphatic vessels, stained with PROX1 (red) and NRP2 (cyan), were wider in caliber (B, C, and E) in E14.5 Fat4 ${ }^{A L E C}$ embryos compared with littermate control embryos. No significant difference was observed in vessel branch points (B, $\mathbf{E}$, and $\mathbf{F}$ ). Whole-mount immunostaining of E18.5 mesenteries with PROX1 (cyan) revealed reduced numbers of PROX1 ${ }^{\text {hi }}$ valves in Fat4 ${ }^{\triangle L E C}$ mesenteric lymphatic vessels (H, J, and $\left.\mathbf{K}\right)$. Fat4 ${ }^{\triangle L E C}$ mesenteric lymphatic valve-forming cells (PROX ${ }^{\text {hi }}$ cells, cyan) failed to polarize and had less laminin- $\alpha 5$ (LAMA5, red) than did the control counterparts ( $\mathbf{I}$ and $\mathbf{L}$ ). Data indicate the mean $\pm S E M$. $n=5$ control embryos and $n=8$ Fat $^{\triangle L L C C}$ embryos ( 3 independent E14.5 litters) (C and F); $n=4$ control embryos and $n=5$ Fat4 $4^{\Delta L E C}$ embryos ( 4 independent E18.5 litters) (J). ${ }^{* *} P<0.01$, by 2-tailed Student's $t$ test. Scale bars: $2.5 \mathrm{~mm}$ (A and D), $100 \mu \mathrm{m}$ (B and E), $250 \mu \mathrm{m}$ ( $\mathbf{H}$ and $\mathbf{K})$, and $25 \mu \mathrm{m}$ ( $\mathbf{I}$ and $\mathbf{L})$. Tam, tamoxifen.

phatic endothelial progenitor cells upon their exit from the cardinal veins at E11.5. Whole-mount immunostaining of E11.5 embryos and measurement of the nuclear length/ width ratio in LECs migrating away from the cardinal veins revealed that LEC nuclei were significantly less elliptical and therefore less polarized in Fat $4^{\Delta / \Delta}$ embryos compared with littermate controls (Figure $4, \mathrm{~J}-\mathrm{N}$ ), demonstrating that polarity was disrupted from the onset of lymphangiogenesis in the embryo.

Alterations in cell polarity are also important for the cellular rearrangements and collective cell migration crucial for the formation of valve leaflets during lymphatic vessel valve morphogenesis (32). For this reason, we assessed LEC polarity in prospective valve endothelial cells of the mesenteric collecting lymphatic vessels at E18.5. As observed in actively sprouting and migrating lymphatic vessels, the nuclear ellipticity of valve endothelial cells was significantly reduced in Fat4 $4^{4 / \Delta}$ embryos (Figure 4, O-Q), demonstrating their failure to polarize appropriately. Together, these data demonstrate that Fat4 is an important regulator of LEC polarity, both in cells within actively sprouting vessels and in cells within developing valves. Defective polarity would be predicted to result in the increase in lymphatic vessel width observed in the skin of Fat4-deficient mice at E14.5; instead of endothelial cells dividing in a polarized fashion to extend the length of vessels, nonoriented cell division would result in random distribution of dividing cells and increased vessel width. To determine whether defective cell polarity arises because of a LEC-autonomous requirement for Fat4, we quantified nuclear ellipticity in the dermal lymphatic vasculature of E14.5 control and Fat $4^{4 L E C}$ embryos. These analyses revealed significantly reduced nuclear ellipticity and aberrant cell polarity in Fat4 ${ }^{\triangle L E C}$ lymphatic vessels, confirming that FAT4 within LECs is responsible for coordinating polarity (Supplemental Figure 4). Consistent with our observations in the lymphatic vasculature, Fat4 has previously been demonstrated to be required for the 

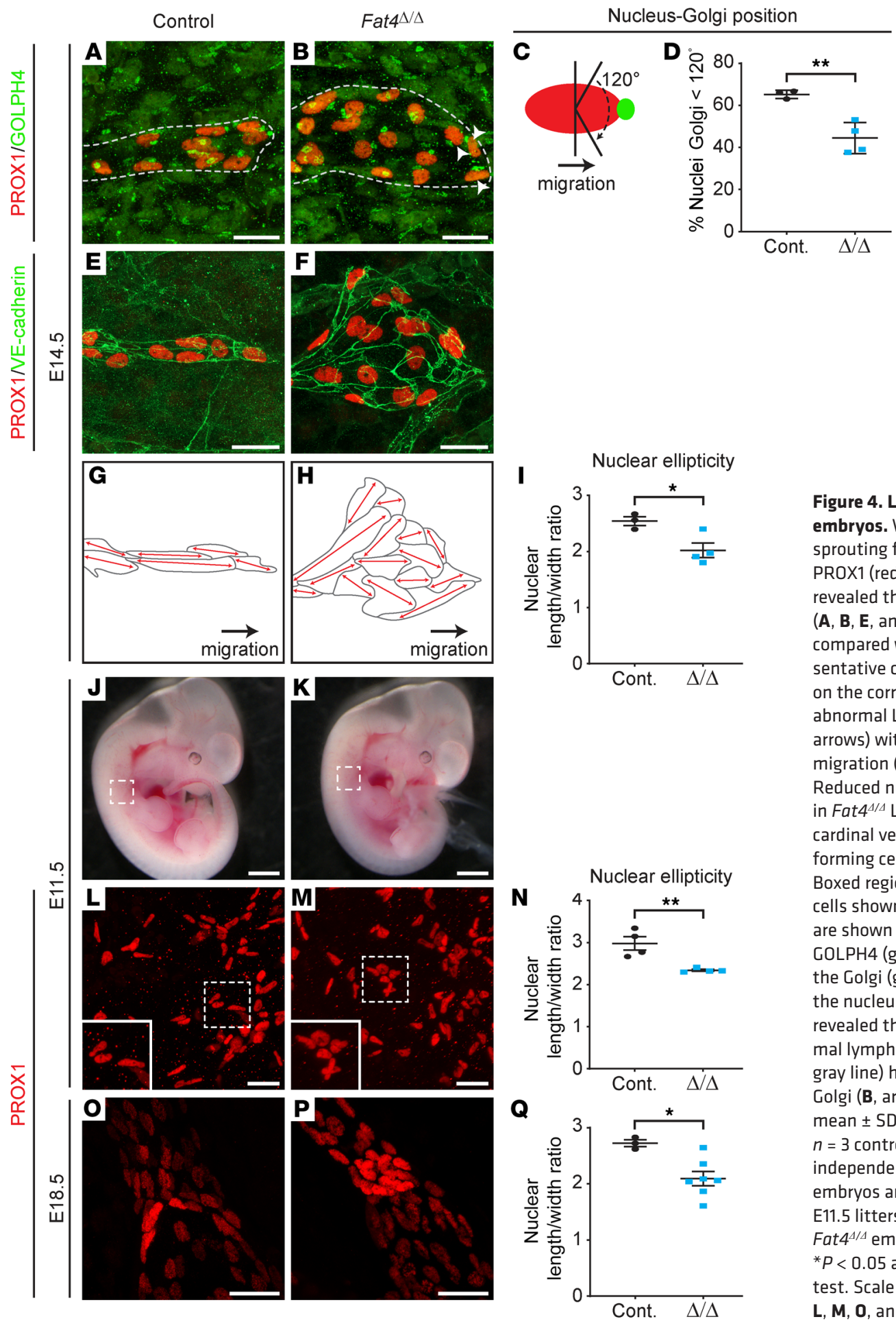

Figure 4. LEC polarity is impaired in Fat4 ${ }^{4 / 4}$ embryos. Whole-mount immunostaining of the sprouting front in E14.5 dorsal skins stained for PROX1 (red) and GOLPH4 or VE-cadherin (green) revealed that Fat $4^{\Delta / \Delta}$ LECs were more rounded

(A, B, E, and F), with reduced nuclear ellipticity compared with littermate control LECs (I). Representative cell shape schematics ( $\mathbf{G}$ and $\mathbf{H}$ ), based on the corresponding vessels in $\mathbf{E}$ and $\mathbf{F}$, highlight abnormal LEC elongation axes (red double-headed arrows) with respect to the direction of vessel migration (black arrows) in Fat4 ${ }^{4 / 4}$ embryos. Reduced nuclear ellipticity was also observed in Fat4 ${ }^{4 / \Delta}$ LECs (PROX1, red) sprouting from the cardinal vein at E11.5 (J-N) and PROX1hi valveforming cells in the mesentery at E18.5 (0-Q). Boxed regions in $\mathbf{J}$ and $\mathbf{K}$ reflect the location of the cells shown in $\mathbf{L}$ and $\mathbf{M}$. Boxed regions in $\mathbf{L}$ and $\mathbf{M}$ are shown at higher magnification in the insets. GOLPH4 (green, $\mathbf{A}$ and $\mathbf{B}$ ) staining highlighting the Golgi (green, $\mathbf{C}$ ), normally located ahead of the nucleus (red, C) in a $120^{\circ}$ arc in migrating cells, revealed that Fat4 ${ }^{\Delta / \Delta}$ LECs in E14.5 sprouting dermal lymphatic vessels (outlined with the dashed gray line) had reduced forward positioning of the Golgi (B, arrowheads, and D). Data indicate the mean \pm SD (D) or the mean \pm SEM $(\mathbf{I}, \mathbf{N}$, and $\mathbf{Q})$. $n=3$ control embryos and $n=4$ Fat $4^{4 / 4}$ embryos (4 independent E14.5 litters) ( $\mathbf{D}$ and $\mathbf{~}$ ); $n=4$ control embryos and $n=4$ Fat $4^{4 / 4}$ embryos ( 3 independent E11.5 litters) (N); $n=3$ control embryos and $n=7$ Fat $4^{4 / 4}$ embryos (3 independent E18.5 litters) (Q)). ${ }^{*} P<0.05$ and ${ }^{* *} P<0.01$, by 2 -tailed Student's $t$ test. Scale bars: $1 \mathrm{~mm}$ (J and $\mathbf{K}), 25 \mu \mathrm{m}$ (A, B, E, F, $\mathbf{L}, \mathbf{M}, \mathbf{O}$, and $\mathbf{P}$ ). oriented cell division important for kidney tubule elongation and cochlear extension during development (25).

Analysis of Hippo pathway activity in Fat4-deficient cells. To determine whether alterations in Hippo pathway activity might also contribute to the lymphatic vascular defects observed in Fat4-deficient mice, we first assessed cell proliferation in the lymphatic vasculature of E14.5 Fat $4^{4 / 4}$ embryos. To this end, we performed immunostaining of E14.5 dorsal skin with antibodies against the mitotic marker phospho-histone $\mathrm{H} 3$ (PH3) and the
LEC surface marker neuropilin 2 (NRP2). These analyses revealed no significant difference in the number of mitotic LECs relative to vessel area in Fat4 $4^{4 / \Delta}$ embryos compared with littermate controls (Supplemental Figure 5, A-C). We next investigated Hippo pathway activity in primary hLECs in vitro following siRNA-mediated FAT4 knockdown. FAT4 siRNA-mediated knockdown of FAT4 in hLECs was efficient (Supplemental Figure 6, A and B), resulting in undetectable FAT4 protein levels 48 hours after treatment. In selected tissue contexts, loss of Fat4 function has been suggest- 


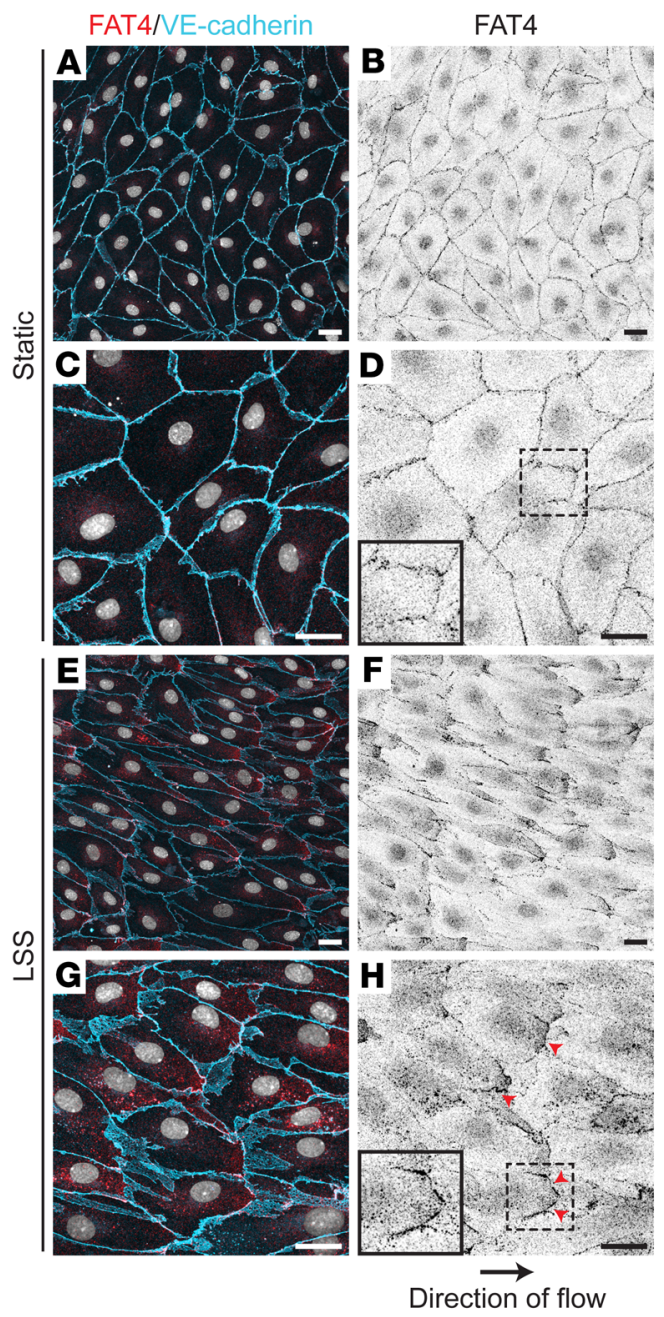

ed to facilitate the nuclear entry of YAP (28-30), enabling YAP interaction with transcriptional cofactors including TEA domain (TEAD) family members and subsequent induction of Hippo pathway target genes including ankyrin repeat domain 1 (ANKRD1), cellular communication network factor 1 (CCN1, also known as CYR61) (40), and CCN2 (4), culminating in cell proliferation. In experiments performed in primary hLECs isolated from 3 independent donors and cultured in complete media, we observed no significant changes in ANKRD1 or CCN1 levels in FAT4-deficient cells. Although CTGF levels were reduced (Supplemental Figure $6 \mathrm{C})$, together these data suggest that there was no major impact on Hippo pathway activity as a result of reduced FAT4 levels. We next assessed Hippo pathway activity in control and FAT4-deficient primary hLECs in response to VEGF-C. To this end, the ratio of cytoplasmic phosphorylated YAP ( $\mathrm{p}$-YAP) compared with total YAP was quantified across a time course of VEGF-C treatment in control and FAT4 siRNA-treated hLECs. No significant changes in the p-YAP/YAP ratio were observed in hLECs following VEGF-C treatment for up to 60 minutes, suggesting that YAP is not dephosphorylated and translocated to the nucleus to activate proliferation in response to VEGF-C. Correspondingly, we observed no differences in the p-YAP/YAP ratio between control and FAT4-deficient cells following VEGF-C treatment (Supplemental Figure 6, D and E). Concordant with these data, VEGF-C was not a major prolif-
Figure 5. FAT4 is polarized in the direction of flow in hLECs. Under static conditions, FAT4 protein (red, A, C, E, and G; black, B, D, F, and H) was localized in a punctate pattern at cell junctions in hLECs (A-D). In response to laminar shear stress (LSS) (4 dynes/( $\left.\mathrm{cm}^{2}\right)$, FAT4 was redistributed (arrowheads) and polarized in the direction of flow (E-H). VE-cadherin (cyan) and DAPI (white) demarcate the cell junctions and nuclei, respectively (A, C, E, and $\mathbf{G})$. Scale bars: $25 \mu \mathrm{m}$ (D and $\mathbf{H})$. Insets depict enlarged images of the boxed regions in $\mathbf{D}$ and $\mathbf{H}$.

erative stimulus for hLECs, and there was no difference in the proliferation of FAT4 siRNA-treated versus control siRNA-treated hLECs stimulated with VEGF-C (Supplemental Figure 7A). Together, these data strongly suggest that the primary mechanism underlying the increased lymphatic vessel caliber in Fat4-deficient mice is defective cell polarity.

FAT4 controls LEC polarity in response to flow. To further investigate the nature of the defective polarity observed in Fat4-deficient LECs in vivo, we first assessed the localization of FAT4 in primary hLECs cultured in vitro, both in static conditions and following exposure to laminar flow, a stimulus that promotes LEC elongation in the direction of flow (42). In static conditions, FAT4 was observed in a uniformly distributed, punctate pattern at cellcell junctions (Figure 5, A-D, and Supplemental Figure 8). Intriguingly, we found that FAT4 was redistributed in a polarized pattern following the exposure of hLECs to laminar flow (Figure 5, E-H). We next assessed whether flow-induced changes in hLEC polarity were dependent on FAT4. In static conditions, we observed no substantial differences in the size or shape of FAT4-deficient cells compared with their control counterparts (Figure 6, A, C, and $\mathrm{E}-\mathrm{I})$. In contrast, in response to laminar flow, FAT4-deficient cells failed to polarize appropriately and instead remained circular (Figure 6, B and D-I). Moreover, the surface area of FAT4-deficient cells following exposure to flow was significantly increased compared with that of FAT4-deficient cells in static conditions (Figure 6, C-E), a factor that could potentially contribute to the phenotype of enlarged vessels observed in Fat4-deficient mice. These data are the first to our knowledge to reveal a role for FAT4 in mechanotransduction and demonstrate an important role for FAT4 in coordinating LEC polarity in response to flow.

Analysis of VEGF-C-initiated VEGFR3 signaling in FAT4-deficient LECs. To explore the possibility that FAT4 is a component of the ADAMTS3/CCBE1/VEGF-C/VEGFR3 signaling axis, given that mutations in ADAMTS3, CCBE1, and FAT4 all underlie Hennekam syndrome, we investigated whether modulation of FAT4 levels affected the processes of VEGF-C cleavage and activation or VEGF-C-mediated VEGFR3 signal transduction. Toward the first of these goals, we performed coimmunoprecipitation assays to determine whether the large extracellular domain (ECD) of FAT4 could be coprecipitated with VEGF-C, CCBE1, or ADAMTS3, all of which are secreted proteins. No detectible association of the FAT4 ECD with any of these proteins was observed (Supplemental Figure 9A). We next assessed whether the extracellular domain of FAT4 modulates ADAMTS3- and CCBE1-mediated proteolytic processing of pro-VEGF-C to fully active VEGF-C. These assays revealed that the processing of proVEGF-C to its fully cleaved, bioactive form was not affected by the addition of the recombinant FAT4 extracellular domain (Sup- 


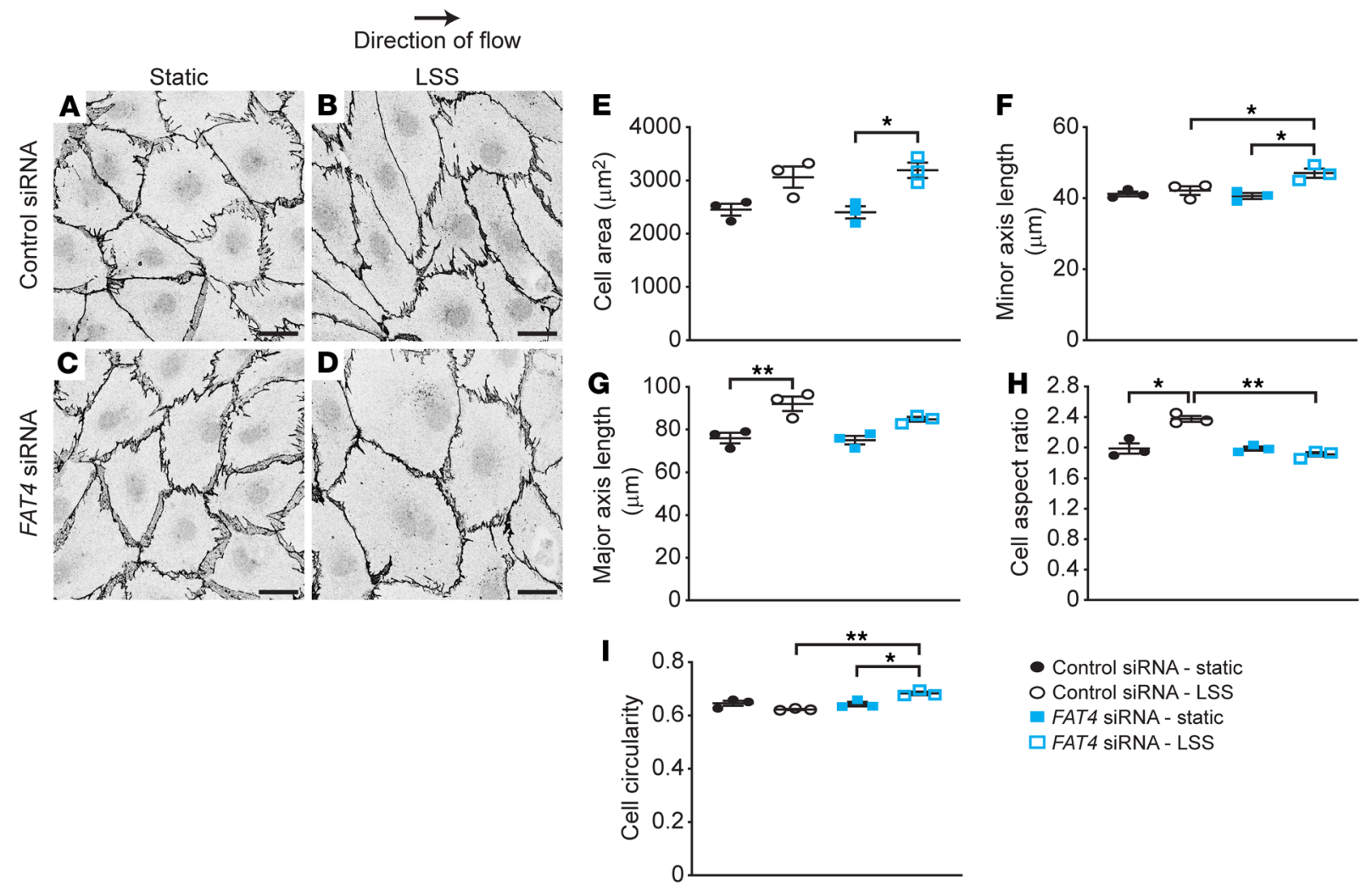

Figure 6. FAT4-deficient hLECs fail to polarize in response to laminar shear stress. Under static conditions, control siRNA- and FAT4 siRNA-treated hLECs were indistinguishable from one another. In response to LSS (4 dynes/cm²), FAT4 siRNA-treated hLECs became larger and more rounded and failed to elongate in the direction of flow (A-I). Representative images using VE-cadherin staining to demarcate the cell outline are shown (A-D). Scale bars: $25 \mu \mathrm{m}$. Data indicate the mean \pm SEM. $n=3 .{ }^{*} P<0.05$ and ${ }^{* *} P<0.01$, by 1-way ANOVA with Tukey's test for multiple comparisons.

plemental Figure 9B), suggesting that FAT4 does not influence the rate or efficiency of VEGF-C activation.

To investigate the possibility that FAT4 might regulate VEGF-Cinitiated VEGFR3 signal transduction, we first assessed the activity of signaling pathways known to be induced in LECs as a consequence of VEGF-C-mediated VEGFR3 activation $(14,43)$. To this end, we quantified VEGF-C-driven phosphorylation of the serine/ threonine kinase AKT and the MAP kinase ERK in Fat4 siRNAtreated primary embryonic mLECS and FAT4 siRNA-treated primary adult hLECs compared with control-treated cells across a 60 -minute time course. We performed the experiments in 3 independent batches of embryonic dermal mLECs, each isolated from 17 to 24 embryos, and in 4 independent batches of hLECs (isolated from different donors). Immunoblotting of cell lysates harvested before VEGF-C treatment and 15, 30, or 60 minutes after VEGF-C treatment revealed that both AKT and ERK phosphorylation were significantly elevated in Fat4-deficient mLECs 15 and 30 minutes after VEGF-C treatment (Supplemental Figure 10, A-D). Interestingly, adult hLECs showed a trend toward elevated AKT activity following VEGF-C treatment (Supplemental Figure 10, E and G) compared with control-treated cells, though this difference was not statistically significant when data were combined from multiple experiments performed across 4 independent batches of hLECs. In contrast, we observed no substantial differences in ERK activity in FAT4-deficient hLECs compared with controls following VEGF-C stimulation (Supplemental Figure 10, F and H). When we compared the responses of FAT4-deficient hLECs isolated from different donors with VEGF-C treatment (Supplemental Figure 10, I and J), we noted that 2 donors had significantly elevated AKT activity in FAT4 siRNA-treated cells, whereas 2 did not (Supplemental Figure 10I). These data reveal that variation exists among donors and highlight the importance of assessing gene function across multiple independent batches of cells. Our data also demonstrate that Fat4-deficient embryonic mLECs had elevated responses to VEGF-C, possibly reflecting increased sensitivity of embryonic versus adult LECs to this key signaling pathway.

To assess whether altered VEGFR3 levels, localization, or activation might underlie the elevated AKT activity observed in the 2 most responsive batches of FAT4-deficient hLECs, we examined total VEGFR3 levels in control siRNA- and FAT4 siRNA-treated hLECs before and after VEGF-C treatment. Immunoblotting of cell lysates revealed no substantial differences in the total levels of VEGFR3 present in FAT4-deficient cells compared with levels in control cells before or after VEGF-C treatment, though, as expected, VEGFR3 levels progressively decreased over a time course of VEGF-C treatment (Supplemental Figure 11, A and B). We next assessed the levels of VEGFR3 present on the cell surface and available to bind VEGF-C in FAT4-deficient compared with control-treated hLECs. In this case, flow cytometry using an antibody that binds the extracellular domain of VEGFR3 was performed to quantify the amount of cell-surface VEGFR3 in control and FAT4-deficient cells before and after VEGF-C treatment 
(Supplemental Figure 11C). We observed no significant differences in cell-surface VEGFR3 levels between FAT4-deficient and control-treated cells and, in both cases, VEGFR3 appeared to be internalized following VEGF-C binding, suggesting that the initial phases of signal transduction were not affected as a result of FAT4 deficiency (Supplemental Figure 11C). To investigate the activation status of VEGFR3 following VEGF-C treatment, we assessed VEGFR3 phosphorylation following 15 minutes of treatment with VEGF-C by immunoprecipitating VEGFR3 and then immunoblotting the precipitated receptor with a pan-phosphotyrosine antibody. These experiments revealed that consistently more VEGFR3 could be immunoprecipitated from FAT4-deficient cells than from control cells (Supplemental Figure 11D). Likewise, elevated levels of tyrosine-phosphorylated VEGFR3 were detected in FAT4-deficient cells compared with levels in control cells (Supplemental Figure 11D). Intriguingly, although more VEGFR3 was immunoprecipitated in FAT4-deficient cells, the ratio of tyrosine-phosphorylated VEGFR3 to total VEGFR3 immunoprecipitated was not significantly altered between FAT4-deficient and control cells (Supplemental Figure 11E), suggesting that FAT4 deficiency does not impact initial VEGFR3 activation.

The intracellular domain of FAT4 interacts with VEGFR3. In considering mechanisms by which FAT4 deficiency might regulate AKT and ERK activity downstream of VEGFR3 activation, we reasoned that a physical interaction between FAT4 and VEGFR3 could potentially influence the downstream signaling of VEGFR3 following receptor internalization. To investigate this further, we performed coimmunoprecipitation assays to assess whether VEGFR3 could associate with the intracellular domain (ICD) of FAT4 when ectopically expressed in human embryonic kidney 293 (HEK293) cells. Immunoblotting of the immunoprecipitated FAT4 ICD revealed a robust interaction between the FAT4 ICD and VEGFR3, both in the absence and presence of VEGF-C (Supplemental Figure 11F). Control experiments confirmed the specificity of immunoprecipitations (Supplemental Figure 11G). These data demonstrate that FAT4 and VEGFR3 have the capacity to physically associate and, therefore, that FAT4 has the potential to regulate signal transduction downstream of VEGFR3 activation. Moreover, this interaction might explain why more VEGFR3 could be immunoprecipitated from FAT4-deficient cells, should VEGFR3 be more accessible to the antibody used for VEGFR3 pulldown in the absence of FAT4.

FAT4 regulation of $L E C$ responses to $V E G F-C$. To investigate the biological impact that elevated, VEGF-C-initiated signaling might have on FAT4-deficient LECs, we assessed the responses of FAT4-deficient primary hLECs to both VEGF-C and complete media, using established proliferation, sprouting, and migration assays. All experiments were performed in at least 3 independent batches of hLECs (isolated from different donors). In the case of proliferation, which we have previously shown is not primarily driven by VEGF-C (44), we observed no significant differences between control and FAT4 siRNA-treated hLECs grown for 48 hours in basal media (Endothelial Cell Basal Medium 2 [EBM-2], $2 \%$ FBS), basal media containing VEGF-C (100 ng/mL), or Microvascular Endothelial Cell Growth Medium-2 SingleQuots complete media (EGM-2MV) (Supplemental Figure 7A). These data are consistent with our analyses of proliferation in vivo, in which we observed no difference in the mitotic index of LECs between Fat $4^{4 / \Delta}$ embryos and their control counterparts (Supplemental Figure 5, A-C). Chemotactic migration toward basal media (EBM2, 0.5\% FBS), basal media containing VEGF-C (100 ng/mL), and complete media was assessed in Transwell assays. Mirroring the scenario in Fat4-mutant mice, we observed no differences in the migration of FAT4-deficient hLECs compared with control hLECs in any of these conditions (Supplemental Figure 7B). We next investigated the capacity of FAT4-deficient hLECs to sprout in response to basal media, VEGF-C, and complete media in 3D spheroid assays. VEGF-C is a prominent sprouting and migration stimulus for LECs $(12,44)$. We observed no significant differences in the number of sprouts extended in basal media, VEGF-C, or complete media (Supplemental Figure 7, C and D) between FAT4-deficient and control hLECs, mirroring the lack of difference in the number of filopodia in the sprouting dermal lymphatic vasculature of E14.5 Fat4 ${ }^{4 / \Delta}$ and control embryos (Supplemental Figure 5, D-F). Intriguingly, FAT4-deficient cells exhibited a trend toward increased sprouting in basal media and VEGF-C compared with control cells. Though this increased sprouting capacity of FAT4-deficient hLECs in vitro potentially reflects the elevated levels of VEGF-C-initiated AKT activity observed in these cells, we think it unlikely that changes in the magnitude of VEGF-Cinitiated signaling contributed significantly to the lymphatic phenotype of $\mathrm{Fat}^{4 / \Delta}$ mice, given that we did not observe changes in lymphatic vessel sprouting or proliferation in vivo.

\section{Discussion}

Here, we identify a crucial, LEC-autonomous role for the atypical cadherin FAT4 during lymphatic vessel morphogenesis, revealing that FAT4 orchestrates lymphangiogenesis primarily by regulating LEC polarity. We demonstrate that Fat4 is required to coordinate LEC polarity from the initiation of lymphangiogenesis in the mouse embryo and that defects in LEC polarity result in the aberrant morphology and disrupted valve development that are characteristic of the lymphatic vasculature in Fat4-mutant embryos. In addition to defective polarity, FAT4-deficient embryonic mLECs exhibited elevated AKT and ERK activity downstream of VEGF-C-induced VEGFR3 phosphorylation in vitro. Though we believe the degree to which this role of FAT4 contributes to the lymphatic vascular phenotype of Fat4-deficient mice is probably minimal, our data provide a potential link between FAT4 and the VEGFR3 signaling pathway, suggesting a mechanism by which mutations in FAT4, $C C B E 1$, and ADAMTS3 underlie Hennekam syndrome.

Previous analyses of Fat4-null mice demonstrated that FAT4 regulates $\mathrm{PCP}$ in tissues including the kidney, neural tube, inner ear, and skeletal tissue (25-27), whereas defects in neural and nephron progenitor cells and cardiomyocytes have been attributed to aberrant Hippo pathway activity as a result of Fat4 loss of function (28-30). Our data suggest that in the lymphatic endothelium, Fat4 functions predominantly via regulation of LEC polarity rather than Hippo pathway activity. PCP in both invertebrates and vertebrates is regulated via 2 major pathways: the "core" pathway consisting of frizzled, dishevelled, Diego, Van Gogh, prickle, and flamingo components in Drosophila (FZD3, -6; DVL1, -2, -3; VANGL2; Prickle1, -2; and CELSR1, -2, -3 in mammals) and the FAT/ Dachsous pathway consisting of FAT, Dachsous, and Four-jointed 
in Drosophila (FAT4/DCHS1/FJX1 in mammals) (45). The mechanisms by which FAT4 transmits signals to downstream components of the cell polarity machinery to mediate cell polarization are not completely understood, but genetic interactions between FAT4 and components of both the FAT/Ds (FAT1) and core pathways (VANGL2) have been documented $(25,46,47)$. The recent demonstration of a requirement for both Celsr1 and Vangl2 in lymphatic vessel valve morphogenesis (32) suggests the intriguing possibility that FAT4 might cooperate with these core PCP proteins to coordinate valve endothelial cell polarity. Further dissection of the molecular mechanisms via which Fat4 coordinates LEC polarity will be explored in future work.

How do the FAT4 mutations found in Hennekam syndrome impact FAT4 function? Though we have demonstrated here that Fat4 is crucial to coordinate LEC polarity during lymphatic vessel morphogenesis in vivo and in response to flow in vitro, the mechanisms by which Hennekam syndrome mutations alter FAT4 activity to cause lymphatic vascular dysfunction remain to be established. To date, the homozygous and compound heterozygous mutations reported in Hennekam syndrome are largely missense mutations located in the cadherin repeats of the extracellular domain of FAT4 that are predicted to be deleterious by SIFT and PolyPhen algorithms (21). To further explore the potential consequences of Hennekam syndrome mutations on FAT4 structure in silico, we substituted Hennekam syndrome mutations into the corresponding, highly conserved regions of the E-cadherin ectodomain, for which high-resolution crystal structures have been established (48). Hennekam syndrome mutations Phe475Leu, Glu486Gln, and Glu2375Lys, all of which result in changes to residues highly conserved between FAT4 and E-cadherin, revealed that these mutations would be predicted to disrupt calcium coordination (48), a crucial factor in cadherin function. Previous work from Tsukasaki and colleagues reported that the huge extracellular domains of FAT4 and DCHS1 adopt a structure compatible with the confines of intercellular spaces due to their substitution of amino acids in the linker regions between cadherin repeats responsible for calcium binding (49). Loss of calcium binding capacity resulted in the adoption of sharp, hairpin-like bends, predicted to enable FAT4 and DCHS1 to adopt a compact spatial organization (49). On the basis of this work, the disruption of calcium-coordinating residues due to Hennekam syndrome mutations might be expected to result in hairpin-like bends that could have severe consequences on the structure of the FAT4 extracellular domain, potentially affecting the binding of FAT4 to DCHS1 and/or other ligands or coreceptors. Alternatively, Hennekam mutations might affect the ability of FAT4 to transduce flow- or shear-induced signal transduction. VEGFR3 is a key component of the VEGFR2-VEGFR3-VE-cadherin mechanosensory complex in endothelial cells and is important for coordinating changes in cell orientation that occur in response to shear stress $(50,51)$. Our identification of an interaction between VEGFR3 and FAT4 raises the possibility that FAT4 might also regulate the capacity of VEGFR3 to transduce flow-mediated signals. Although our data demonstrate a link between FAT4 and the VEGF-C/VEGFR3 pathway in primary LECs in vitro, we believe that the lymphatic vascular defects observed in Fat $4^{\Delta / \Delta}$ mice are largely explained by defective LEC polarity. It will be intriguing to investigate the con- sequences of FAT4 mutations found in Hennekam syndrome for both LEC polarity and VEGF-C initiated signaling via VEGFR3.

Why does loss of FAT4 function not have the same impact as loss of CCBE1 or ADAMTS3 function on VEGF-C/VEGFR3 signal transduction, given that mutations in all 3 genes underlie Hennekam syndrome? Lymphangiectasia, presenting as dilation of lymphatic vessels and often associated with lymphedema (52), is not only a feature of Hennekam syndrome but has been reported in human lymphatic dysplasia syndromes including Noonan syndrome (53), caused by hyperactivation of the RAS/MAPK signaling pathway and excessive lymphangiogenesis (54). It is likely that mechanisms including defective LEC polarity, defective valve development, and hyperplasia of the lymphatic vasculature all result in lymphangiectasia. The lymphangiectasia reported in patients with Hennekam syndrome is a prominent feature of Fat4-null mice and is probably caused by defective cell polarity. Of interest, Fat4 deletion in mice results in a less-severe lymphatic phenotype than does deletion of either Ccbe1 or Adamts3, both of which essentially mirror Vegfc loss of function. In the patients with Hennekam syndrome studied to date, the onset of lymphedema in patients with $C C B E 1$ has been reported from birth, whereas lymph edema can occur at a later point in Hennekam syndrome caused by FAT4 mutations, suggesting that CCBE1 mutations are more deleterious with respect to their effect on embryonic lymphangiogenesis. It is also possible that in Hennekam syndrome, yet-to-bedescribed phenotypic differences exist that are caused by CCBE1, ADAMTS3, and FAT4 mutations. Intriguingly, lymphedema has been reported in only 1 patient with Van Maldergem syndrome to date (55). Van Maldergem syndrome shares features of Hennekam syndrome, including periventricular neuronal heterotopia, and is caused by mutations in FAT4 or DCHS1 (29). The report of only 1 patient with a DCHS1 mutation who had obvious lymphedema (55) suggests that DCHS1 mutations are yet to be identified in Hennekam syndrome or, alternatively, that mutations in additional components of the FAT4/DCHS1 pathway may also underlie Hennekam syndrome. Variation in patient phenotype has been reported even between patients carrying the same GATA2 mutation, which causes lymphedema in some patients and hematological or immune dysfunction in others (56).

While this manuscript was in preparation, a brief report was published describing a requirement for Fat4 and Dchs1 in lymphatic vessel valve morphogenesis (38). This study concurs with ours in documenting a role for Fat4 in the control of LEC polarity that is important for valve development, but did not dissect the LECautonomous requirement for these proteins or describe their roles in earlier phases of lymphangiogenesis before valve development. Our study is also the first to our knowledge to document a potential link between FAT4 and the VEGF-C/VEGFR3 signaling pathway.

In conclusion, we document a crucial, LEC-autonomous role for Fat4 in the control of LEC polarity. We demonstrate that orchestration of polarity is crucial for building functional lymphatic vessels; disruption of LEC polarity due to Fat4 loss of function results in aberrant lymphatic vessel morphology and defective lymphatic vessel valve morphogenesis. We also provide the first evidence, to our knowledge, demonstrating that FAT4 is important for mechanotransduction and, in particular, for transducing flow-induced signals in LECs. It will be fascinating to further 
dissect the mechanisms by which FAT4 transduces mechanical signals in endothelial cells. Identification of the molecular components important for regulating LEC polarity, mechanotransduction, and VEGFR3 signaling via Fat4 will form the basis of our future studies, as will further investigation into the mechanisms by which Hennekam syndrome mutations impact FAT4 function. Answers to these questions will provide important insight into how alterations in lymphatic vessel architecture result in human lymphatic vascular diseases including Hennekam syndrome.

\section{Methods}

Mouse studies. Fat4 ${ }^{f / f l}$ (25), Prox1-CreERT2 (36), and CMV-Cre (34) mice have been previously described. All mouse lines were backcrossed onto a C57BL/6J background. Adult female mice were subjected to timed pregnancies, scored by the presence of vaginal plugs, with 9:00 am on the day of plug detection designated 0.5 days postcoitum. To generate Fat4-null mice, Fat $4^{f / f l}$ mice were crossed with $\mathrm{CMV}$-Cre mice to generate $\mathrm{Fat}^{+/ / 4}$ mice, which were crossed to yield Fat $^{4 / \Delta}$ mice. For excision of floxed alleles using Prox1-CreERT2, 20 $\mathrm{mg} / \mathrm{mL}$ tamoxifen (MilliporeSigma) was dissolved in peanut oil containing $10 \%$ (v/v) ethanol. Pregnant mice were injected intraperitoneally with $2.5 \mathrm{mg}$ per $25 \mathrm{~g}$ body weight at the indicated time points. Littermate control embryos were either $\mathrm{Fat}_{4}^{+/+}$or $\mathrm{Fat}^{\mathrm{fl} / \mathrm{fl} l}$ depending on the Cre deleter strain used.

Antibodies. For immunofluorescence staining, the following primary antibodies were used: goat anti-human PROX1 (AF2727; R\&D Systems), rat anti-mouse FOXC2 (57), rabbit anti-GOLPH4 (ab28049; abcam), rabbit anti-mouse laminin $\alpha 5$ (58), goat anti-human neuropilin 2 (AF2215; R\&D Systems), rabbit neuropilin 2 XP (3366; Cell Signaling Technology), rat anti-endomucin (V.7C7, sc-65495; Santa Cruz Biotechnology), rat anti-mouse CD31 (102502; BioLegend), rat anti-mouse CD144 (550548; BD Biosciences), goat anti-mouse VE-cadherin (AF1002; R\&D Systems), anti- $\alpha$-smooth muscle actinCy3 antibody (C6198; MilliporeSigma), rabbit anti-FAT4 (HPA052819; MilliporeSigma), and rabbit PH3 (Ser10) (06-570; MilliporeSigma). Alexa Fluor-conjugated antibodies (Life Technologies, Thermo Fisher Scientific) were used for visualization. Rabbit anti-AKT (9272; Cell Signaling Technology), rabbit anti-p-AKT (Thr308) (4056; Cell Signaling Technology), rabbit anti-p44/42 MAPK (ERK1/2, 4695; Cell Signaling Technology), rabbit anti-p-p44/42 MAPK (Thr202/Tyr204) (4370; Cell Signaling Technology), goat anti-FAT4 (P-17, sc-161577; Santa Cruz Biotechnology), mouse anti- $\beta$-actin (A5441; MilliporeSigma), mouse anti-phosphotyrosine (p-Tyr-100, 9411; Cell Signaling Technology), mouse anti-FLT4 (G-3, sc-365748; Santa Cruz Biotechnology), goat anti-mouse VEGFR3 (AF743; R\&D Systems), mouse anti-YAP (H-9, sc-271134; Santa Cruz Biotechnology), rabbit anti-p-YAP (Ser127) (4911; Cell Signaling Technology), normal rabbit IgG (sc-2027; Santa Cruz Biotechnology), rabbit anti-GATA2 (H-116X, sc-9008; Santa Cruz Biotechnology), mouse anti-V5-HRP (46-0708; Invitrogen, Thermo Fisher Scientific), mouse anti-FLAG M2-HRP (A8592; MilliporeSigma), mouse anti-HA.11 epitope tag (MMS-101P; BioLegend), and rabbit anti-DYKDDDDK tag antibody (2368; Cell Signaling Technology) were used for immunoblotting and immunoprecipitation experiments.

Immunostaining. For cryopreserved sections and whole-mount staining of skin, embryos were fixed in $4 \%$ paraformaldehyde (PFA) overnight at $4^{\circ} \mathrm{C}$. Mesenteries were fixed in $4 \%$ PFA for 10 minutes or 1 hour at room temperature (RT). Whole-mount tissues and sections were immunostained as previously described (59). Images were captured at RT using either a Carl Zeiss LSM 700 Axio Observer Z1 confocal microscope equipped with $405 \mathrm{~nm}, 488 \mathrm{~nm}, 555 \mathrm{~nm}$, and $639 \mathrm{~nm}$ lasers, or a Carl Zeiss LSM 800 Axio Observer 7 confocal microscope with Airyscan, equipped with $405 \mathrm{~nm}, 488 \mathrm{~nm}, 561 \mathrm{~nm}$, and $640 \mathrm{~nm}$ lasers. Images were compiled using ZEN 2.5 (blue edition; Zeiss) and Adobe Photoshop CC (version 20.0.1) software.

Quantification of lymphatic vessel parameters. All lymphatic vessel parameters were quantified in at least 3 embryos per genotype across a minimum of 3 independent litters. The sample number for each experiment is specified in the figure legends. Dermal lymphatic vessel width $(\mu \mathrm{m})$ and branch points were quantified within $1.28 \mathrm{~mm}$ of the sprouting front on both sides of the dorsal midline using Image (NIH) (60) as previously described (59). The number of mesenteric lymphatic vessel valves was counted along 5 to 12 spokes per mesentery and normalized to the spoke length ( $\mathrm{mm}$ ), measured using Image (60).

PH3-positive LECs were counted in dermal lymphatic vessels within $640 \mu \mathrm{m}$ of the sprouting front on both sides of the dorsal midline (3 images from each side of the midline, totaling 6 images per embryo) and normalized to the lymphatic vessel area $\left(\mathrm{mm}^{2}\right)$, measured using ImageJ (60). Filopodial counts were performed using the same 6 images and normalized to the 100- $\mu \mathrm{m}$ lymphatic vessel length at the sprouting front.

The Golgi position in relation to nuclei was scored (either within or outside the $120^{\circ}$ frontal arc) in cells within $160 \mu \mathrm{m}$ of the sprouting front on both sides of the dorsal midline. Six fields of view (FOV) per E14.5 embryo were counted, equating to 50-70 nuclei. To determine nuclear ellipticity (ratio of nuclear length/width), nuclear width and length were measured using Image (60). For E11.5 analyses, 50-80 nuclei per embryo were measured, for E14.5 analyses 40-130 nuclei per embryo were measured, and for E18.5 analyses, nuclei were measured in 20-50 PROX $1^{\text {hiFOXC }} 2^{\text {hi }}$ valve-forming endothelial cells per mesentery.

Cell culture. Primary adult human dermal microvascular LECs (HMVEC-dLyAd) were purchased from Lonza and are referred to here as hLECs. All in vitro experiments were performed in at least 3 independent batches of hLECs (isolated from different donors; lot numbers 7F3304, 0000254463, 4F3029, and 4F3037). Recombinant VEGF-C from R\&D Systems (2179-VC-025) or ReliaTech (R20-015) was used for assays. Primary embryonic mLECs (mLECs) were isolated from E16.5 mouse dermis as previously described (44). hLECs and mLECs were cultured in EBM-2 (Lonza) supplemented with EGM2MV (Lonza). HEK293 cells (a gift from Stuart Pitson, Centre for Cancer Biology, Adelaide, Australia) were maintained in DMEM with high glucose containing $10 \%$ FBS.

Transfection. mLECs were transfected using Lipofectamine RNAiMAX Transfection Reagent (Invitrogen, Thermo Fisher Scientific) according to the manufacturer'sinstructions 24 and 48 hours after seeding using FAT4 Silencer Select Predesigned siRNA (s116449; Thermo Fisher Scientific). hLECs were transfected using Lipofectamine RNAiMAX or Lipofectamine 2000 Transfection Reagent (Invitrogen, Thermo Fisher Scientific) according to the manufacturer's instructions and with FAT4 Silencer Select Predesigned siRNA (s35967; Thermo Fisher Scientific), FAT4 MISSION esiRNA (EHU069061; MilliporeSigma), or GATA2 Silencer Select Predesigned siRNA (s5596; Thermo Fisher Scientific). Silencer Select Negative Control No. 1 siRNA (4390844; Thermo Fisher Scientific) or EGFP MISSION esiRNA negative control (EHUEGFP; MilliporeSigma) was used as a control treatment for the respective experiments. 
ChIP-Seq analysis. Sheared chromatin from 10 million cell equivalents was immunoprecipitated with $15 \mu \mathrm{g}$ rabbit anti-GATA2 antibody (H-116X, sc-9008; Santa Cruz Biotechnology). ChIP-Seq library preparation and sequencing were carried out at the Australian Cancer Research Foundation (ACRF) Cancer Genomics Facility, Centre for Cancer Biology (Adelaide, Australia) as previously described (61). ChIP-Seq data have been deposited in the European Nucleotide Archive (ENA) under the accession number PRJEB9436 (http://www. ebi.ac.uk/ena/data/view/PRJEB9436).

RNA analysis. Total RNA was isolated from cells using TRIzol (Invitrogen, Thermo Fisher Scientific) according to the manufacturer's instructions. For investigation of mRNA levels, RNA was reverse transcribed using the QuantiTect Reverse Transcription Kit (QIAGEN). Real-time RT-PCR was performed in triplicate using $\mathrm{RT}^{2}$ SYBR Green ROX qPCR Mastermix (QIAGEN) and run on a Corbett Research Rotor-Gene 6000 real-time rotary analyzer (QIAGEN) using the primers listed in Supplemental Table 1. Data were analyzed using RotorGene Q Series software (version 2.3.1; QIAGEN). All data were normalized to the housekeeping gene $A L A S 1$, as previously described (62).

$I S H$. RNA ISH was performed as previously described (63), using Fat4 digoxigenin-labeled riboprobes (25). Probes were hybridized to cryopreserved E14.5 WT 10- $\mu \mathrm{m}$ sections. Probe specificity was confirmed using a corresponding sense probe. Immunostaining for PROX1 was performed following ISH as previously described (59). Whole embryo bright-field scans were captured using 3DHISTECH Pannoramic 250 Flash II. PROX1 costaining was acquired using confocal microscopy as detailed above. Images were compiled using ZEN 2.5 (blue edition; Zeiss) and Adobe Photoshop CC (version 20.0.1) software.

Immunoblotting and immunoprecipitation. To assess endogenous FAT4 protein levels, transfected cells were harvested in T-PER Tissue Protein Extraction Reagent (Thermo Fisher Scientific) supplemented with Halt Protease and Phosphatase Inhibitor Cocktail (Thermo Fisher Scientific). After addition of $4 \times$ Laemmli sample buffer (200 mM Tris, pH 6.8, 400 mM DTT, $8 \%$ [w/v] SDS, $40 \%$ glycerol, bromophenol blue), protein extracts were heated at $95^{\circ} \mathrm{C}$ for 3 minutes. Samples were resolved by SDS-PAGE (Bio-Rad Criterion XT 3\%-8\% Tris-Acetate Precast Protein Gels), transferred onto PVDF membranes (PerkinElmer), and blotted with respective primary antibodies. Immunoblots were visualized using ECF reagent (GE Healthcare) or Immun-Star AP substrate (Bio-Rad) on a Typhoon FLA 9000 (GE Healthcare) or ChemiDoc MP Imaging system (Bio-Rad) and quantified using ImageQuant TL 1D (version 8.1; GE Healthcare) or Image Lab (Bio-Rad) software.

To assess VEGF-C-initiated signaling and Hippo pathway activity, transfected cells were serum starved for 16 hours in EBM-2 supplemented with 0.05\% AlbuMAX (Gibco, Thermo Fisher Scientific) (mLECs) or 0.5\% FBS (hLECs) and stimulated with VEGF-C (200 ng/ $\mathrm{mL}$ for $\mathrm{mLECs}$ or $100 \mathrm{ng} / \mathrm{mL}$ for hLECs) for the designated durations. Cells were then harvested in $4 \times$ Laemmli sample buffer $(200 \mathrm{mM}$ Tris, pH 6.8, 400 mM DTT, 8\% [w/v] SDS, 40\% glycerol, bromophenol blue) and heated at $95^{\circ} \mathrm{C}$ for 7 minutes. For immunoblotting, samples were resolved by SDS-PAGE (Bio-Rad Mini-PROTEAN TGX StainFree 4\%-20\% Precast Protein Gels), transferred onto PVDF membranes (PerkinElmer), and blotted with the respective primary antibodies. Immunoblots were visualized as detailed above.

To assess VEGFR3 phosphorylation, hLECs were transfected with control or FAT4 siRNA, serum starved for 16 hours with EBM-2 sup- plemented with $0.5 \%$ FBS, and stimulated with $100 \mathrm{ng} / \mathrm{mL}$ VEGF-C for 15 minutes. Cells were harvested in ice-cold PBS and collected by centrifugation $\left(200 \times g\right.$ for 10 minutes at $\left.4^{\circ} \mathrm{C}\right)$. Cell pellets were lysed in lysis buffer containing $20 \mathrm{mM}$ Tris, $\mathrm{pH} 7.5,150 \mathrm{mM} \mathrm{NaCl}$, and 1\% IGEPAL CA-630 (MilliporeSigma) supplemented with Halt Protease and Phosphatase Inhibitor Cocktail (Thermo Fisher Scientific). Lysates were sonicated and centrifuged, and the protein concentrations were estimated using a BCA Protein Assay Kit (Pierce, Thermo Fisher Scientific) according to the manufacturer's instructions. To immunoprecipitate VEGFR3, $125 \mu \mathrm{g}$ of the respective cell lysate was incubated with $1.25 \mu$ g mouse anti-Flt4 (G-3, sc-365748; Santa Cruz Biotechnology) and antibody-antigen complexes were precipitated with Dynabeads Protein G (Life Technologies, Thermo Fisher Scientific). Immunoprecipitates were washed with lysis buffer, resuspended in Laemmli sample buffer, and heated at $95^{\circ} \mathrm{C}$ for 5 minutes. Immunoblotting was performed as detailed above.

To analyze protein interactions via coimmunoprecipitation, HEK293 cells were cotransfected with FLAG-tagged FAT4 intracellular domain (NM_183221, residues 4527-4981) in pCMV6-Entry (OriGene) and VEGFR3 (NM_182925) in pcDNA3.1(+) expression vector (Thermo Fisher Scientific) or empty vector using Lipofectamine 2000 (Invitrogen, Thermo Fisher Scientific) according to the manufacturer's instructions. Cotransfected cells were lysed in lysis buffer, sonicated, and centrifuged. To immunoprecipitate the FAT4 intracellular domain, cell lysates prepared from cells ectopically expressing FAT4 intracellular domain and VEGFR3 or empty vector were incubated with rabbit anti-DYKDDDDK tag antibody (2368; Cell Signaling Technology) or normal rabbit IgG (sc-2027; Santa Cruz Biotechnology), and antibody-antigen complexes were precipitated with Dynabeads Protein G (Life Technologies, Thermo Fisher Scientific). To immunoprecipitate VEGFR3, lysates prepared from cells ectopically expressing FAT4 intracellular domain and VEGFR3 were incubated with goat anti-mouse VEGFR3 (AF743; R\&D Systems) or control IgG and antibody-antigen complexes precipitated with Dynabeads Protein G (Life Technologies, Thermo Fisher Scientific). Immunoprecipitates were washed with lysis buffer, resuspended in Laemmli sample buffer, and heated at $95^{\circ} \mathrm{C}$ for 5 minutes. Immunoblotting was performed as detailed above.

FAT4 cloning and protein expression. The extracellular domain of human FAT4 (FAT4-EC) was cloned by amplifying multiple overlapping fragments of FAT4 by PCR, using cDNA reverse transcribed from RNA harvested from hLECs (Lonza) as a template, and assembled into the pCMV6-AC-HA vector (Origene) using NEBuilder HiFi DNA Assembly (E2621; New England BioLabs). HEK293T cells were plated at a density of $3 \times 10^{5}$ cells per $10 \mathrm{~cm}^{2}$, and epitope-tagged cDNA constructs were transfected into these cells using FuGENE6 Transfection Reagent (E2691; Promega). Unless otherwise indicated, conditioned media were collected 72 hours after transfection. Untransfected HEK293T conditioned media were collected and used as control supernatant. Immunoblotting was performed using anti-V5-HRP (46-0708; Invitrogen, Thermo Fisher Scientific), anti-FLAG M2-HRP (A8592; MilliporeSigma), and anti-HA.11 epitope tag (MMS-101P; BioLegend) antibodies and developed with ECL reagent (Thermo Fisher Scientific).

Immunoprecipitation of FAT4 with VEGF-C, CCBE1, ADAMTS3. Conditioned supernatants were harvested and mixed for 18 hours at $4^{\circ} \mathrm{C}$ before addition of anti-HA-conjugated magnetic beads (11201D; Thermo Fisher Scientific and sc-7392; Santa Cruz Biotechnology). Beads were washed 3 times using Opti-MEM (Thermo Fisher Scien- 
tific), and bound proteins were eluted in sample buffer and analyzed by immunoblotting.

Pro-VEGF-C processing. Conditioned supernatant from HEK293 cells transfected with VEGF-C-VHD-FLAG-CT-HA, CCBE1-V5, FAT4-EC-HA, or untransfected controls was mixed at a 1:1:1 ratio at $37^{\circ} \mathrm{C}$. Samples were collected at various time points and analyzed by immunoblotting to detect the state of VEGF-C processing.

In vitro proliferation. Transfected hLECs were seeded in 96-well microplates at $5 \times 10^{3}$ cells per well with 5 replicates for each treatment. Cells were cultured for 24 hours before a 16- hour starvation in EBM-2 supplemented with 0.5\% FBS. Cells were fixed with 4\% PFA and stained with hematoxylin immediately following serum starvation (to normalize the number of cells plated) or after a 24-hour stimulation with basal media (EBM-2 supplemented with $2 \% \mathrm{FBS}$ ), basal media containing $100 \mathrm{ng} / \mathrm{mL}$ VEGF-C, or EGM-2MV complete media (Lonza). Cells were quantified using ImageQuant TL Colony Counting (version 8.1; GE Healthcare) software. The proliferative index represents the number of cells relative to the initial number of cells plated (represented as 1.0).

Transwell migration assay. Transwell assays to assess chemotactic cell migration were performed as previously described (64), with the additional step of coating the top and bottom surfaces of Transwell membranes with $50 \mu \mathrm{g} / \mathrm{mL}$ fibronectin (FN) (Roche). Cell migration toward basal media (EBM-2 supplemented with 0.5\% FBS), basal media containing $100 \mathrm{ng} / \mathrm{mL}$ VEGF-C or EGM-2MV was assessed. Data represented as percentage of cells migrated through the membrane relative to initial number of cells plated $\left(1 \times 10^{4}\right.$ cells $)$.

Sprouting assay. 3D sprouting assays were performed as previously described (64).

In vitro flow assay. Transfected hLECs $\left(2 \times 10^{5}\right.$ cells $)$ were seeded in FN-coated $\left(50 \mu \mathrm{g} / \mathrm{mL}\right.$; Roche) $\mu$-Slide I ${ }^{0.8}$ Luer channel slides (ibidi) and cultured for 24 hours in EGM-2MV to allow cells to reach confluence. Cells were then subjected to laminar flow ( 4 dynes $\left./ \mathrm{cm}^{2}\right)$ using a yellow-green perfusion set (ibidi) combined with the Quad Pump System (ibidi), or were maintained under static conditions, for 48 hours. Cells were fixed for 10 minutes in 4\% PFA, blocked in PBS containing $1 \%$ BSA and $0.3 \%$ Triton X-100, and stained as previously described (65). Nuclei were visualized using DAPI Fluoromount G (ProSciTech). Images were acquired using confocal microscopy as detailed above. Cell parameters were measured using Image (60) and VE-cadherin staining to demarcate cell outlines. A total of 250 cells across 5 FOV were analyzed per condition, per experiment. The cell aspect ratio is defined as the major axis length relative to the minor axis length (i.e., length/width). For cell circularity $\left(4 \pi \times\right.$ area/perimeter $\left.{ }^{2}\right)$, a value of 1.0 indicates a perfect circle, whereas a value approaching 0 indicates a more elongated shape.

Flow cytometry. To assess surface levels of VEGFR3, hLECs were transfected with control or FAT4 esiRNA, serum starved for 16 hours with EBM-2 supplemented with 0.5\% FBS, and stimulated with 100 $\mathrm{ng} / \mathrm{mL}$ VEGF-C for 15 minutes. Immediately following treatment, cells were dissociated using TrypLE Express Enzyme (Thermo Fisher Scientific), pelleted, resuspended in $200 \mu \mathrm{L}$ ice-cold PBS supplemented with $5 \%$ FBS, and incubated on ice for 20 minutes. To detect VEGFR3, $1 \mu \mathrm{L}$ APC-conjugated mouse anti-human VEGFR3 antibody (FAB3492A, lot LGB0517071; R\&D Systems) was added, incubated on ice for 20 minutes, and washed by centrifugation $(200 \times g$ for 5 minutes at $4^{\circ} \mathrm{C}$ ) with $4 \mathrm{~mL}$ ice-cold PBS supplemented with $5 \%$ FBS. Cells were fixed in 2\% PFA and analyzed using an LSRFortessa cell analyzer (BD Biosciences). Data analysis and visualization were performed using FlowJo software (version 10.6.1; BD Biosciences).

Statistics. Unless stated otherwise, $P$ values were calculated using the 2-tailed Student's $t$ test. In vitro flow data were analyzed with GraphPad Prism (version 8.0.1; GraphPad Software) using a 1-way ANOVA with Tukey's test for multiple comparisons. $P$ values of less than 0.05 were considered statistically significant.

Study approval. Experiments using mice were approved by and conducted in accordance with guidelines of the South Australian (SA) Pathology/Central Adelaide Local Health Network (CALHN) Animal Ethics Committee, the University of South Australia Animal Ethics Committee, and the Australian National Health and Medical Research Council (NHMRC).

\section{Author contributions}

KLB, DLS, GAS, JK, AO, and LL designed and performed experiments, analyzed data, and prepared figures. NM and LS generated and contributed antibodies. HM generated and contributed mice. KLB, DLS, and NLH wrote the manuscript. NLH, HM, MLK, and $\mathrm{BMH}$ conceptualized the study and analyzed data. All authors edited the manuscript. KLB performed the initial experiments of the study and was therefore assigned the first authorship position.

\section{Acknowledgments}

We thank Chris Brown and staff at the SA Pathology Animal Facility and UniSA Core Animal Facility for animal husbandry. This study used the services of the Australian Phenomics Network Histopathology and Organ Pathology Service (University of Melbourne, Parkville, Australia) and the SAHMRI Histology Slide Scanning Service (Adelaide, Australia). Confocal microscopy and flow cytometry were performed at the Detmold Imaging and Flow Cytometry Facility (UniSA and SA Pathology, Adelaide, Australia). This work was supported by grants from the NHMRC (APP1061365 and APP1146352, to NLH), the Australian Research Council (ARC) (DP150103110, to BMH and NLH), and the Hospital Research Foundation (to NLH). NLH was supported by an ARC Future Fellowship (FT130101254).

Address correspondence to: Natasha Harvey, Centre for Cancer Biology, UniSA and SA Pathology, GPO Box 2471, Adelaide, South Australia, 5001, Australia. Phone: 61.8.8302.7835; Email: natasha.harvey@unisa.edu.au.
1. Hennekam RC, et al. Autosomal recessive intestinal lymphangiectasia and lymphedema, with facial anomalies and mental retardation. Am J Med Genet. 1989;34(4):593-600.

2. Bellini C, et al. Hennekam syndrome presenting as nonimmune hydrops fetalis, congenital chy- lothorax, and congenital pulmonary lymphangiectasia. Am JMed Genet A. 2003;120A(1):92-96.

3. Van Balkom ID, et al. Lymphedema-lymphangiectasia-mental retardation (Hennekam) syndrome: a review. Am JMed Genet. 2002;112(4):412-421.
4. Alders M, et al. Mutations in CCBE1 cause generalized lymph vessel dysplasia in humans. Nat Genet. 2009;41(12):1272-1274.

5. Hogan BM, et al. Ccbe1 is required for embryonic lymphangiogenesis and venous sprouting. Nat Genet. 2009;41(4):396-398. 
6. Bos FL, et al. CCBE1 is essential for mammalian lymphatic vascular development and enhances the lymphangiogenic effect of vascular endothelial growth factor-C in vivo. Circ Res. 2011;109(5):486-491.

7. Le Guen L, et al. Ccbe1 regulates Vegfc-mediated induction of Vegfr3 signaling during embryonic lymphangiogenesis. Development. 2014;141(6):1239-1249.

8. Jeltsch M, et al. CCBE1 enhances lymphangiogenesis via A disintegrin and metalloprotease with thrombospondin motifs-3-mediated vascular endothelial growth factor-C activation. Circulation. 2014;129(19):1962-1971.

9. Brouillard P, et al. Loss of ADAMTS3 activity causes Hennekam lymphangiectasia-lymphedema syndrome 3. Hum Mol Genet. 2017;26(21):4095-4104.

10. Zheng W, Aspelund A, Alitalo K. Lymphangiogenic factors, mechanisms, and applications. J Clin Invest. 2014;124(3):878-887.

11. Secker GA, Harvey NL. VEGFR signaling during lymphatic vascular development: From progenitor cells to functional vessels. Dev Dyn. 2015;244(3):323-331.

12. Karkkainen MJ, et al. Vascular endothelial growth factor $\mathrm{C}$ is required for sprouting of the first lymphatic vessels from embryonic veins. Nat Immunol. 2004;5(1):74-80.

13. Joukov V, et al. A novel vascular endothelial growth factor, VEGF-C, is a ligand for the Flt4 (VEGFR-3) and KDR (VEGFR-2) receptor tyrosine kinases. EMBO J. 1996;15(2):290-298.

14. Mäkinen T, et al. Isolated lymphatic endothelial cells transduce growth, survival and migratory signals via the VEGF-C/D receptor VEGFR-3. EMBO J. 2001;20(17):4762-4773.

15. Balboa-Beltran E, et al. A novel stop mutation in the vascular endothelial growth factor-C gene (VEGFC) results in Milroy-like disease. JMed Genet. 2014;51(7):475-478.

16. Gordon K, et al. Mutation in vascular endothelial growth factor-C, a ligand for vascular endothelial growth factor receptor-3, is associated with autosomal dominant milroy-like primary lymphedema. Circ Res. 2013;112(6):956-960.

17. Ferrell RE, et al. Hereditary lymphedema: evidence for linkage and genetic heterogeneity. Hum Mol Genet. 1998;7(13):2073-2078.

18. Gordon K, et al. FLT4/VEGFR3 and Milroy disease: novel mutations, a review of published variants and database update. Hum Mutat. 2013;34(1):23-31.

19. Irrthum A, Karkkainen MJ, Devriendt K, Alitalo K, Vikkula M. Congenital hereditary lymphedema caused by a mutation that inactivates VEGFR3 tyrosine kinase. Am J Hum Genet. 2000;67(2):295-301.

20. Mendola A, et al. Mutations in the VEGFR3 signaling pathway explain $36 \%$ of familial lymphedema. Mol Syndromol. 2013;4(6):257-266.

21. Alders M, et al. Hennekam syndrome can be caused by FAT4 mutations and be allelic to Van Maldergem syndrome. Hum Genet. 2014;133(9):1161-1167.

22. Hoeng JC, et al. Identification of new human cadherin genes using a combination of protein motif search and gene finding methods. J Mol Biol.
2004;337(2):307-317.

23. Rock R, Schrauth S, Gessler M. Expression of mouse dchs1, fjx1, and fat-j suggests conservation of the planar cell polarity pathway identified in Drosophila. Dev Dyn. 2005;234(3):747-755.

24. Sopko R, McNeill H. The skinny on Fat: an enormous cadherin that regulates cell adhesion, tissue growth, and planar cell polarity. Curr Opin Cell Biol. 2009;21(5):717-723.

25. Saburi S, et al. Loss of Fat 4 disrupts PCP signaling and oriented cell division and leads to cystic kidney disease. Nat Genet. 2008;40(8):1010-1015.

26. Zakaria S, et al. Regulation of neuronal migration by Dchs1-Fat4 planar cell polarity. Curr Biol. 2014;24(14):1620-1627.

27. Mao Y, et al. Dchs1-Fat4 regulation of polarized cell behaviours during skeletal morphogenesis. Nat Commun. 2016;7:11469.

28. Das A, et al. Stromal-epithelial crosstalk regulates kidney progenitor cell differentiation. Nat Cell Biol. 2013;15(9):1035-1044.

29. Cappello S, et al. Mutations in genes encoding the cadherin receptor-ligand pair DCHS1 and FAT4 disrupt cerebral cortical development. Nat Genet. 2013;45(11):1300-1308.

30. Ragni CV, et al. Amotl1 mediates sequestration of the Hippo effector Yap1 downstream of Fat 4 to restrict heart growth. Nat Commun. 2017;8:14582.

31. Mao Y, et al. Characterization of a Dchs1 mutant mouse reveals requirements for Dchs1-Fat4 signaling during mammalian development. Development. 2011;138(5):947-957.

32. Tatin F, et al. Planar cell polarity protein Celsr1 regulates endothelial adherens junctions and directed cell rearrangements during valve morphogenesis. Dev Cell. 2013;26(1):31-44.

33. Gonzalez-Garay ML, et al. A novel mutation in CELSR1 is associated with hereditary lymphedema. Vasc Cell. 2016;8:1.

34. Schwenk F, Baron U, Rajewsky K. A cre-transgenic mouse strain for the ubiquitous deletion of loxPflanked gene segments including deletion in germ cells. Nucleic Acids Res. 1995;23(24):5080-5081.

35. Bazigou $\mathrm{E}$, et al. Integrin-alpha9 is required for fibronectin matrix assembly during lymphatic valve morphogenesis. Dev Cell. 2009;17(2):175-186.

36. Srinivasan RS, et al. Lineage tracing demonstrates the venous origin of the mammalian lymphatic vasculature. Genes Dev. 2007;21(19):2422-2432.

37. Durst R, et al. Mutations in DCHS1 cause mitral valve prolapse. Nature. 2015;525(7567):109-113.

38. Pujol F, et al. Dachsous1-Fat4 signaling controls endothelial cell polarization during lymphatic valve morphogenesis - brief report. Arterioscler Thromb Vasc Biol. 2017;37(9):1732-1735.

39. Coxam B, et al. Pkd1 regulates lymphatic vascular morphogenesis during development. Cell Rep. 2014;7(3):623-633.

40. Stein C, et al. YAP1 exerts its transcriptional control via TEAD-mediated activation of enhancers. PLoS Genet. 2015;11(8):e1005465.

41. Zhao B, et al. TEAD mediates YAP-dependent gene induction and growth control. Genes Dev. 2008;22(14):1962-1971.

42. Sabine A, et al. Mechanotransduction, PROX1, and FOXC2 cooperate to control connexin 37 and calcineurin during lymphatic-valve formation.
Dev Cell. 2012;22(2):430-445.

43. Deng Y, Zhang X, Simons M. Molecular controls of lymphatic VEGFR3 signaling. Arterioscler Thromb Vasc Biol. 2015;35(2):421-429.

44. Kazenwadel J, Secker GA, Betterman KL, Harvey NL. In vitro assays using primary embryonic mouse lymphatic endothelial cells uncover key roles for FGFR1 signalling in lymphangiogenesis. PLoS ONE. 2012;7(7):e40497.

45. Davey CF, Moens CB. Planar cell polarity in moving cells: think globally, act locally. Development. 2017;144(2):187-200

46. Badouel C, et al. Fat1 interacts with Fat4 to regulate neural tube closure, neural progenitor proliferation and apical constriction during mouse brain development. Development. 2015;142(16):2781-2791.

47. Saburi S, Hester I, Goodrich L, McNeill H. Functional interactions between Fat family cadherins in tissue morphogenesis and planar polarity. Development. 2012;139(10):1806-1820.

48. Harrison OJ, et al. The extracellular architecture of adherens junctions revealed by crystal structures of type I cadherins. Structure. 2011;19(2):244-256.

49. Tsukasaki Y, et al. Giant cadherins Fat and Dachsous self-bend to organize properly spaced intercellular junctions. Proc Natl Acad Sci USA 2014;111(45):16011-16016.

50. Baeyens N, et al. Vascular remodeling is governed by a VEGFR3-dependent fluid shear stress set point. Elife. 2015;4:e04645.

51. Coon BG, et al. Intramembrane binding of VE-cadherin to VEGFR2 and VEGFR3 assembles the endothelial mechanosensory complex. JCell Biol. 2015;208(7):975-986.

52. Bellini C, Hennekam RC. Clinical disorders of primary malfunctioning of the lymphatic system. Adv Anat Embryol Cell Biol. 2014;214:187-204

53. Joyce $\mathrm{S}$, et al. The lymphatic phenotype in Noonan and cardiofaciocutaneous syndrome. Eur J Hum Genet. 2016;24(5):690-696.

54. Deng Y, Atri D, Eichmann A, Simons M. Endothelial ERK signaling controls lymphatic fate specification. J Clin Invest. 2013;123(3):1202-1215.

55. Mansour S, et al. Van Maldergem syndrome: further characterisation and evidence for neuronal migration abnormalities and autosomal recessive inheritance. Eur J Hum Genet. 2012;20(10):1024-1031.

56. Spinner MA, et al. GATA2 deficiency: a protean disorder of hematopoiesis, lymphatics, and immunity. Blood. 2014;123(6):809-821.

57. Furumoto TA, et al. Notochord-dependent expression of MFH1 and PAX1 cooperates to maintain the proliferation of sclerotome cells during the vertebral column development. Dev Biol. 1999;210(1):15-29.

58. Ringelmann B, et al. Expression of laminin alpha1, alpha2, alpha4, and alpha5 chains, fibronectin, and tenascin-C in skeletal muscle of dystrophic 129ReJ dy/dy mice. Exp Cell Res. 1999;246(1):165-182

59. Betterman KL, Harvey NL. Histological and morphological characterization of developing dermal lymphatic vessels. Methods Mol Biol. 2018;1846:19-35

60. Abramoff MD, Magalhaes PJ, Ram SJ. Image processing with ImageJ. Biophotonics Int 
2004;11(7):36-42.

61. Kazenwadel J, et al. GATA2 is required for lymphatic vessel valve development and maintenance. J Clin Invest. 2015;125(8):2979-2994.

62. Livak KJ, Schmittgen TD. Analysis of relative gene expression data using real-time quantitative PCR and the 2(-Delta Delta C(T)) method. Meth- ods. 2001;25(4):402-408.

63. Schwarz Q, et al. Vascular endothelial growth factor controls neuronal migration and cooperates with Sema3A to pattern distinct compartments of the facial nerve. Genes Dev. 2004;18(22):2822-2834

64. Kartopawiro J, et al. Arap3 is dysregulated in a mouse model of hypotrichosis-lymphedema-telan- giectasia and regulates lymphatic vascular development. Hum Mol Genet. 2014;23(5):1286-1297.

65. Betterman KL, et al. Remodeling of the lymphatic vasculature during mouse mammary gland morphogenesis is mediated via epithelialderived lymphangiogenic stimuli. Am J Pathol. 2012;181(6):2225-2238. 